\title{
PROOF OF THE GGS CONJECTURE
}

\author{
TRAVIS SCHEDLER
}

\begin{abstract}
We prove the GGS conjecture [GGS] (1993), which gives a particularly simple explicit quantization of classical $r$-matrices for Lie algebras $\mathfrak{g l}(n)$, in terms of a matrix $R \in \operatorname{Mat}_{n}(\mathbb{C}) \otimes \operatorname{Mat}_{n}(\mathbb{C})$ which satisfies the quantum YangBaxter equation (QYBE) and the Hecke condition, whose quasiclassical limit is $r$. The $r$-matrices were classified by Belavin and Drinfeld in the 1980's in terms of combinatorial objects known as Belavin-Drinfeld triples. We prove this conjecture by showing that the GGS matrix coincides with another quantization from [ESS], which is a more general construction. We do this by explicitly expanding the product from [ESS] using detailed combinatorial analysis in terms of Belavin-Drinfeld triples.
\end{abstract}

\section{Introduction}

In the 1980's, Belavin and Drinfeld classified solutions $r$ of the classical YangBaxter equation (CYBE) for simple Lie algebras $\mathfrak{g}$ satisfying $0 \neq r+r^{21} \in\left(S^{2} \mathfrak{g}\right)^{\mathfrak{g}}$ $[\mathrm{BD}]$. They proved that all such solutions fall into finitely many continuous families and introduced combinatorial objects to label these families, BelavinDrinfeld triples (see Section 1.1). In 1993, Gerstenhaber, Giaquinto, and Schack attempted to quantize such solutions for Lie algebras $\mathfrak{s l}(n)$. As a result, they formulated a conjecture stating that certain explicitly given elements $R_{\mathrm{GGS}} \in$ $\operatorname{Mat}_{n}(\mathbb{C}) \otimes \operatorname{Mat}_{n}(\mathbb{C})$ satisfy the quantum Yang-Baxter equation (QYBE) and the Hecke relation [GGS]. Specifically, the conjecture assigns a family of such elements to any Belavin-Drinfeld triple of type $A_{n-1}$. This conjecture is stated in Section 1.2.

Recently, Etingof, Schiffmann, and the author found an explicit quantization of all $r$-matrices from the Belavin-Drinfeld list. They did so by twisting the coproduct in the Drinfeld-Jimbo quantum group $U_{q}(\mathfrak{g})$. For $\mathfrak{g}=\mathfrak{g l}(n)$, one can evaluate the universal $R$-matrix of the twisted $U_{q}(\mathfrak{g})$ in the vector representation of $U_{q}(\mathfrak{g})$. This gives an element $R_{J}$ of $\operatorname{Mat}_{n}(\mathbb{C}) \otimes \operatorname{Mat}_{n}(\mathbb{C})$ which satisfies the QYBE and the Hecke relation. This element is presented in Section 1.3.

In this paper I show that the elements $R_{J}$ and $R_{\mathrm{GGS}}$ from [ESS] and [GGS] coincide. This proves the GGS conjecture. This is done by explicitly expanding the formula for $R_{J}$ using combinatorial techniques involving Belavin-Drinfeld triples. The proof occupies all of Section 2.

Received March 14, 1999.

Revision received September 19, 2000. 
Remark 1.1. Note that the GGS conjecture was proved in some special cases (the Cremmer-Gervais and orthogonal disjoint cases) by Hodges in [H2] and [H]. The GGS conjecture was proved in some additional cases (orthogonal generalized disjoint) by the author in [S2]. Also, the disjoint case was completed in [S2] by Pavel Etingof and the author.

Remark 1.2. The author actually found the matrix $R_{J}$ with the help of a computer before the general twist given in [ESS] was found. The matrix was constructed to coincide with the GGS matrix in many cases, and motivated in part the general construction given in [ESS]. See [S] for details. Also, many steps of this proof were motivated by and checked with computer programs.

1.1. Belavin-Drinfeld triples. Let $\left(e_{i}\right), 1 \leq i \leq n$, be the standard basis for $\mathbb{C}^{n}$. Let $\Gamma=\left\{e_{i}-e_{i+1}: 1 \leq i \leq n-1\right\}$ be the set of simple roots of $\mathfrak{s l}(n)$. We will use the notation $\alpha_{i} \equiv e_{i}-e_{i+1}$. Let $($,$) denote the inner product on \mathbb{C}^{n}$ having $\left(e_{i}\right)$ as an orthonormal basis.

Definition 1.1. [BD] A Belavin-Drinfeld triple of type $A_{n-1}$ is a triple $\left(T, \Gamma_{1}, \Gamma_{2}\right)$ where $\Gamma_{1}, \Gamma_{2} \subset \Gamma$ and $T: \Gamma_{1} \rightarrow \Gamma_{2}$ is a bijection, satisfying two relations:

(a) $T$ preserves the inner product: $\forall \alpha, \beta \in \Gamma_{1},(T \alpha, T \beta)=(\alpha, \beta)$.

(b) $T$ is nilpotent: $\forall \alpha \in \Gamma_{1}, \exists k \in \mathbb{N}$ such that $T^{k} \alpha \notin \Gamma_{1}$.

Let $\mathfrak{g}=\mathfrak{g l}(n)$ be the Lie algebra of complex $n \times n$ matrices. Let $\mathfrak{h} \subset \mathfrak{g}$ be the subspace of diagonal matrices. Elements of $\mathbb{C}^{n}$ define linear functions on $\mathfrak{h}$ by $\left(\sum_{i} \lambda_{i} e_{i}\right)\left(\sum_{i} a_{i} e_{i i}\right)=\sum_{i} \lambda_{i} a_{i}$. Let $P=\sum_{1 \leq i, j \leq n} e_{i j} \otimes e_{j i}$ be the Casimir element inverse to the standard form on $\mathfrak{g}$. It is easy to see that $P(w \otimes v)=v \otimes w$, for any $v, w \in \mathbb{C}^{n}$. Let $P^{0}=\sum_{i} e_{i i} \otimes e_{i i}$ be the projection of $P$ to $\mathfrak{h} \otimes \mathfrak{h}$.

For any Belavin-Drinfeld triple, consider the following equations for $s \in \mathfrak{h} \wedge \mathfrak{h}$ :

$$
\forall \alpha \in \Gamma_{1},[(\alpha-T \alpha) \otimes 1] s=\frac{1}{2}[(\alpha+T \alpha) \otimes 1] P^{0} .
$$

Belavin and Drinfeld showed that solutions $r \in \mathfrak{g} \otimes \mathfrak{g}$ of the CYBE satisfying $r+r^{21}=P$, up to isomorphism, are given by a discrete datum (the BelavinDrinfeld triple) and a continuous datum (a solution $s \in \mathfrak{h} \wedge \mathfrak{h}$ of (1.1)). We now describe this classification.

For $\alpha=e_{i}-e_{j}$, set $e_{\alpha} \equiv e_{i j}$. Define $|\alpha|=|j-i|$. For any $Y \subset \Gamma$, set $\tilde{Y}=\left\{\alpha \in \operatorname{Span}(Y) \mid \alpha=e_{i}-e_{j}, i<j\right\}$ (the set of positive roots of the subalgebra of $\mathfrak{s l}(n)$ having $Y$ as the set of simple roots). In particular we will often use the notation $\tilde{\Gamma}, \tilde{\Gamma}_{1}, \tilde{\Gamma}_{2}$. We extend $T$ additively to a map $\tilde{\Gamma}_{1} \rightarrow \tilde{\Gamma}_{2}$, i.e. $T(\alpha+\beta)=T \alpha+T \beta$. Whenever $T^{k} \alpha=\beta$ for $k \geq 1$, we say $\alpha \prec \beta$. Clearly $\prec$ is a partial ordering on $\tilde{\Gamma}$. We will also use $\alpha \preceq \beta$ to denote $\alpha \prec \beta$ or $\alpha=\beta$. Suppose $T^{k} \alpha=\beta$ for $\alpha=e_{i}-e_{j}$ and $\beta=e_{l}-e_{m}$. Then there are two possibilities on how $T^{k}$ sends $\alpha$ to $\beta$, since $T$ is an automorphism of the Dynkin diagram. Namely, either $T^{k}\left(\alpha_{i}\right)=\alpha_{l}$ and $T^{k}\left(\alpha_{j-1}\right)=\alpha_{m-1}$, or $T^{k}\left(\alpha_{i}\right)=\alpha_{m-1}$ and $T^{k}\left(\alpha_{j-1}\right)=\alpha_{l}$. In the former case, call $T^{k}$ orientation-preserving on $\alpha$, and 
in the latter, orientation-reversing on $\alpha$. Let

$$
C_{\alpha, \beta}= \begin{cases}1, & \text { if } T^{k} \text { reverses orientation on } \alpha, \\ 0, & \text { if } T^{k} \text { preserves orientation on } \alpha .\end{cases}
$$

Now we define

$$
\begin{gathered}
a=\sum_{\alpha \prec \beta}(-1)^{C_{\alpha, \beta}(|\alpha|-1)}\left(e_{-\alpha} \otimes e_{\beta}-e_{\beta} \otimes e_{-\alpha}\right), \\
r_{s t}=\frac{1}{2} \sum_{i} e_{i i} \otimes e_{i i}+\sum_{\alpha \in \tilde{\Gamma}} e_{-\alpha} \otimes e_{\alpha}, \quad r=s+a+r_{s t},
\end{gathered}
$$

$\left(r_{s t} \in \mathfrak{g} \otimes \mathfrak{g}\right.$ is the standard solution of the CYBE satisfying $r_{s t}+r_{s t}^{21}=P$.) The element $r$ is the solution of the CYBE corresponding to the data $\left(\left(\Gamma_{1}, \Gamma_{2}, T\right), s\right)$. It follows from [BD] that any solution $\tilde{r} \in \mathfrak{g}, \tilde{r}+\tilde{r}^{21}=P$ is equivalent to such a solution $r$ under an automorphism of $\mathfrak{g}$.

1.2. The GGS conjecture. The GGS conjecture suggests a quantization of the matrix $r$ given in (1.4), given by a matrix $R \in \operatorname{Mat}_{n}(\mathbb{C}) \otimes \operatorname{Mat}_{n}(\mathbb{C})$ conjectured to satisfy the quantum Yang-Baxter equation (QYBE), $R^{12} R^{13} R^{23}=$ $R^{23} R^{13} R^{12}$, and the Hecke relation, $(P R-q)\left(P R+q^{-1}\right)=0$. This may be formulated and justified as follows (which is more or less the original motivation).

If we write $R \equiv 1+2 \hbar r+4 \hbar^{2} t\left(\bmod \hbar^{3}\right)$, where $q \equiv e^{\hbar}$, then we can consider the constraints imposed by the QYBE and the Hecke relation modulo $\hbar^{3}$. One may easily check that the QYBE becomes the CYBE for $r$, while the Hecke relation becomes the condition $t+t^{21}=r^{2}$. Since it is not difficult to see that $r^{2}$ is symmetric, the unique symmetric choice for $t$ is $t=\frac{1}{2} r^{2}=\frac{1}{2}\left(s^{2}+\left(a+r_{s t}\right) s+\right.$ $\left.s\left(a+r_{s t}\right)+\varepsilon\right)$ where

$$
\varepsilon=a r_{s t}+r_{s t} a+a^{2} .
$$

Proposition 1.1. There exist unique polynomials $P_{i, j, k, l}$ of the form $x q^{y}\left(q-q^{-1}\right)^{z}, x, y \in \mathbb{C}, z \in\{0,1\}$ such that $\sum_{i, j, k, l} P_{i, j, k, l} e_{i j} \otimes e_{k l} \equiv 1+2 \hbar r+$ $2 \hbar^{2} r^{2}\left(\bmod \hbar^{3}\right)$.

Proof. The proof is easy.

Definition 1.2. Define $R_{\mathrm{GGS}}=\sum_{i, j, k, l} P_{i, j, k, l} e_{i j} \otimes e_{k l}$, with the $P_{i, j, k, l}$ uniquely determined by Proposition 1.1. The matrix $R_{\mathrm{GGS}}$ is called the GGS $R$-matrix.

We will use the notation $x=\sum_{i, j, k, l} x_{i k}^{j l} e_{i j} \otimes e_{k l}$ for elements $x \in \operatorname{Mat}_{n}(\mathbb{C}) \otimes$ $\operatorname{Mat}_{n}(\mathbb{C})$. Define the following matrices:

$$
\tilde{a}=\sum_{i, j, k, l} a_{i k}^{j l} q^{a_{i k}^{j l} \varepsilon_{i k}^{j l}} e_{i j} \otimes e_{k l}, \quad \bar{R}_{\mathrm{GGS}}=R_{s t}+\left(q-q^{-1}\right) \tilde{a},
$$

where $R_{s t}=q \sum_{i} e_{i i} \otimes e_{i i}+\sum_{i \neq j} e_{i i} \otimes e_{j j}+\left(q-q^{-1}\right) \sum_{i>j} e_{i j} \otimes e_{j i}$ is the standard Drinfeld-Jimbo solution to the QYBE, which is a quantization of $r_{s t}$. 
Proposition 1.2. The matrix $R_{G G S}$ equals $q^{s} \bar{R}_{G G S} q^{s}$.

Proof. This is a straightforward computation.

Remark 1.3. We see that $R_{\mathrm{GGS}} \equiv q^{2 r}\left(\bmod \hbar^{3}\right)$, although $R_{\mathrm{GGS}} \neq q^{2 r}$ in general.

Conjecture 1.1. "the GGS conjecture" [GGS] The matrix $R_{G G S}$ satisfies the QYBE and the Hecke relation.

Remark 1.4. It is sufficient to check the QYBE for one value of $s$ since the space of solutions to the homogeneous equation corresponding to (1.1) is exactly the space $\Lambda^{2} \mathfrak{l}$ where $\mathfrak{l} \subset \mathfrak{h}$ is the space of all $x$ such that $(x, \alpha)=(x, T \alpha)$ for any $\alpha \in \Gamma_{1}$. Indeed, it is easy to see that $x \in \mathfrak{l}$ implies $\left[1 \otimes x+x \otimes 1, R_{\mathrm{GGS}}\right]=0$, and it follows that $q^{y} R_{\mathrm{GGS}} q^{y}$ satisfies the QYBE iff $R_{\mathrm{GGS}}$ does, for any $y \in \Lambda^{2} \mathfrak{l}$.

Remark 1.5. Our formulation is from $[\mathrm{GH}]$, correcting misprints. The original formulation in [GGS] is somewhat different. We will write $x_{q^{-1}}$ to denote the matrix $x$ with $q^{-1}$ substituted for $q$. Define $(x \otimes y)^{T}=x^{T} \otimes y^{T}$ where $x^{T}$ is the transpose of $x$, for $x, y \in \operatorname{Mat}_{n}(\mathbb{C})$. Then, the original form of $R_{\mathrm{GGS}}$ can be written as follows:

$$
R=q^{-s}\left(R_{s t}+\left(q^{-1}-q\right) \tilde{a}_{q^{-1}}^{T}\right) q^{-s} .
$$

We have $R_{\mathrm{GGS}}-R_{q^{-1}}^{T}=q^{s}\left(q-q^{-1}\right) P q^{s}=\left(q-q^{-1}\right) P$. Thus, $R_{\mathrm{GGS}}$ satisfies the Hecke relation iff $R$ satisfies the Hecke relation. In this case, we have $P R_{q^{-1}}^{T}=$ $\left(P R_{\mathrm{GGS}}\right)^{-1}$, so $R_{q^{-1}}^{T}=\left(R_{\mathrm{GGS}}^{-1}\right)^{21}$, and thus $R$ satisfies the QYBE iff $R_{\mathrm{GGS}}$ does. Thus, the two formulations are equivalent.

1.3. Passed $T$-pairs and a combinatorial formula for $\varepsilon$. In this section we give a combinatorial formula for $\varepsilon$. First let us introduce some definitions, which will be used in this formula as well as in the proof of the main theorem.

Definition 1.3. A positive $T$-pair is a pair $\left(T^{k} \alpha,-\alpha\right)$ for $k>0$. We define the order to be $\operatorname{Ord}\left(T^{k} \alpha,-\alpha\right)=k$. The set of positive $T$-pairs is denoted $T P_{+}$.

In Section 2, we will also define negative $T$-pairs, but so far we don't need them.

Definition 1.4. If $\alpha=e_{i}-e_{j} \in \tilde{\Gamma}_{1}$ satisfies the property that $T^{k} \alpha=e_{j}-e_{2 j-i}$ for some $k>0$, we say its right-passing order is $k$, and denote it by $P^{r}(\alpha)=k$. If there is no such $k$, say $P O^{r}(\alpha)=\infty$. Similarly define left-passing order $P O^{l}$.

Definition 1.5. A positive $T$-pair $(\beta,-\alpha)$ is right-passed if $\operatorname{Ord}(\beta,-\alpha)>P O^{r}(\alpha)$ and $C_{\alpha, \beta}=C_{\alpha, P O^{r}(\alpha)}$. We say that $(\beta,-\alpha)$ is half right-passed if $\operatorname{Ord}(\beta,-\alpha)=P O^{r}(\alpha)$. Similarly define the left versions, and we denote the sets of all such T-pairs by $P T P_{+}^{r}, H P T P_{+}^{r}, P T P_{+}^{l}$, and $H P T P_{+}^{l}$ (right-passed, half right-passed, left-passed, half left-passed, respectively). The + subscripts indicate positive $T$-pairs. 
Definition 1.6. For a positive $T$-pair $(\beta,-\alpha)$, we define $P^{r}(\beta,-\alpha)$ to be 1 if $(\beta,-\alpha) \in P T P_{+}^{r}, \frac{1}{2}$ if $(\beta,-\alpha) \in H P T P_{+}^{r}$, and otherwise 0 . Similarly define $P^{l}(\beta,-\alpha)$. It will be useful to define symmetric and anti-symmetric versions: $P^{s}=P^{r}+P^{l}, P^{a}=P^{r}-P^{l}$.

This allows us to state a simple combinatorial formula for $\varepsilon$ :

Proposition 1.3. We may rewrite $\varepsilon$ as follows:

$$
\begin{aligned}
& \varepsilon= \\
& -\sum_{\alpha \prec \beta}(-1)^{C_{\alpha, \beta}(|\alpha|-1)}\left[P^{s}(\beta,-\alpha)+C_{\alpha, \beta}(|\alpha|-1)\right]\left(e_{\beta} \otimes e_{-\alpha}+e_{-\alpha} \otimes e_{\beta}\right) .
\end{aligned}
$$

Proof. This is proved in Section 2.1 and also follows from the proof of the main theorem, Theorem 1.2 (see Remark 2.1 for details).

Corollary 1.1. $\bar{R}_{G G S}$ is given as follows:

$$
\begin{array}{r}
\bar{R}_{G G S}=\sum_{\alpha \prec \beta}\left(q-q^{-1}\right)(-1)^{C_{\alpha, \beta}(|\alpha|-1)}\left[q^{-P^{s}(\beta,-\alpha)-C_{\alpha, \beta}(|\alpha|-1)}\left(e_{-\alpha} \otimes e_{\beta}\right)\right. \\
\left.-q^{P^{s}(\beta,-\alpha)+C_{\alpha, \beta}(|\alpha|-1)}\left(e_{\beta} \otimes e_{-\alpha}\right)\right]+R_{s t}
\end{array}
$$

Proof. Clear.

Example 1.1. For a given $n$, there are exactly $\phi(n)$ triples $(\phi$ is the Euler $\phi$-function) in which $\left|\Gamma_{1}\right|+1=|\Gamma|[\mathrm{GG}]$. These are called generalized CremmerGervais triples. These are indexed by $m \in \mathbb{Z}^{+}$, where $\operatorname{gcd}(n, m)=1$, and given by $\Gamma_{1}=\Gamma \backslash\left\{\alpha_{n-m}\right\}, \Gamma_{2}=\Gamma \backslash\left\{\alpha_{m}\right\}$, and $T\left(\alpha_{i}\right)=\alpha_{\operatorname{Res}(i+m)}$, where Res gives the residue modulo $n$ in $\{1, \ldots, n\}$. For these triples, there is a unique $s$ with first component having trace 0 , which is given by $s_{i i}^{i i}=0, \forall i$, and $s_{i j}^{i j}=\frac{1}{2}-\frac{1}{n} \operatorname{Res}\left(\frac{j-i}{m}\right)$ for $i \neq j$ (this is easy to verify directly and is also given in [GG]). With this $s$, $R_{\mathrm{GGS}}$ has a very nice combinatorial formula, which was conjectured by Giaquinto and checked in some cases. We now state and prove this formula.

Proposition 1.4. $R_{G G S}$ is given as follows for generalized Cremmer-Gervais triples:

$$
\begin{aligned}
R_{G G S} & = \\
q^{s} R_{s t} q^{s} & +\sum_{\alpha \prec \beta}\left(q-q^{-1}\right)\left[q^{\frac{-2 \operatorname{Ord}(\beta,-\alpha)}{n}}\left(e_{-\alpha} \otimes e_{\beta}\right)-q^{\frac{2 \operatorname{Ord}(\beta,-\alpha)}{n}}\left(e_{\beta} \otimes e_{-\alpha}\right)\right] .
\end{aligned}
$$

Proof. See Appendix A. 
1.4. The ESS twist and the main theorem. In [ESS], an explicit quantization is given for any classical $r$-matrix described in Section 1.1. This is given by a twist of the standard coproduct on the quantum universal enveloping algebra $U_{q}(\mathfrak{g})$. In particular, in the $n$-dimensional representation, this gives an element $J \in \operatorname{Mat}_{n}(\mathbb{C}) \otimes \operatorname{Mat}_{n}(\mathbb{C})$ so that $R_{J}=q^{s} J^{-1} R_{s t} J^{21} q^{s}$ satisfies the QYBE and the Hecke relation. In fact, $J$ is triangular, i.e. $J=1+N$ and $N=\sum_{\alpha, \beta \in \tilde{\Gamma}} N_{\alpha, \beta} e_{\beta} \otimes e_{-\alpha}$.

Suppose we are given $T$ so that $\max _{x \in T P_{+}} \operatorname{Ord}(x)=m$. Define the following matrices:

$$
\begin{gathered}
J_{k}=1+\sum_{\beta=T^{k} \alpha}(-q)^{-C_{\alpha, \beta}(|\alpha|-1)} q^{P^{a}(\beta,-\alpha)}\left(q-q^{-1}\right) e_{\beta} \otimes e_{-\alpha}, \\
J=\prod_{i=1}^{m} J_{i}, \quad \bar{R}_{J}=J^{-1} R_{s t} J^{21}, \quad R_{J}=q^{s} \bar{R}_{J} q^{s} .
\end{gathered}
$$

Theorem 1.1. [ESS] The element $R_{J}$ satisfies the $Q Y B E$ and the Hecke relation.

Now, we state the main theorem of this paper:

Theorem 1.2. For any given $T$ and $s, R_{J}=R_{G G S}$.

This theorem clearly implies the GGS conjecture.

\section{Proof of the main theorem}

\subsection{T-quadruples.}

Overview 2.1. In this section we will introduce combinatorial objects and arguments which are sufficient to prove the combinatorial formula for $\varepsilon$ (1.8). To do this, we wish to directly expand (1.5). This involves expanding a quadratic expression in terms of the form $e_{\beta} \otimes e_{-\alpha}$ and $e_{-\alpha} \otimes e_{\beta}$ for $\alpha \preceq \beta$. Most of the monomials in the expansion are zero. Thus, the first step is to restrict our attention to those that are not. In this vein, we define compatible $T$-quadruples (Definition 2.9). To further simplify the formula for $\varepsilon$, we will have to show that most of these monomials cancel pairwise. This is accomplished with bijections $\phi, \psi, \psi^{l}$, and $\psi^{r}$ (Definitions 2.13-2.15) between the corresponding $T$-quadruples.

Notation 2.1. For all of section 2 we will not need to refer to the dimension of the representation (formerly $n$ ), so we will reuse $n$ for other purposes.

Definition 2.1. For any subset $Y \subset \Gamma$, define $\bar{Y}=(\tilde{Y} \cup-\tilde{Y})$.

Definition 2.2. Let $T P_{-}=\{(-\alpha, \beta) \in-\tilde{\Gamma} \times \tilde{\Gamma} \mid \alpha \preceq \beta\}$. These will be called negative $T$-pairs. We define $\operatorname{Ord}\left(-\alpha, T^{k} \alpha\right)=k$, and $P^{*}\left(-\alpha, T^{k} \alpha\right)=$ $P^{*}\left(T^{k} \alpha,-\alpha\right)$ for $* \in\{l, r, a, s\}$.

Note 2.1. For negative $T$-pairs, we allow the order to be zero, but not for positive ones! 
Definition 2.3. For $x \in T P$, define $S_{x}=1$ if $x \in T P_{+}$and $S_{x}=-1$ if $x \in T P_{-}$.

Definition 2.4. We define $P T P_{-}^{*}$ and $H P T P_{-}^{*}$ just as in the positive case, but with components in $T$-pairs permuted. Let $P T P^{*}=P T P_{-}^{*} \cup P T P_{+}^{*}$ and $H P T P^{*}=H P T P_{-}^{*} \cup H P T P_{+}^{*}$.

Note 2.2. Note that all passed elements must have positive order, so there is nothing new in the case of negative passed $T$-pairs.

Definition 2.5. For $x=(\beta,-\alpha) \in T P_{+}$, set $x_{l}=\alpha$ and $x_{h}=\beta$ (lower, higher, respectively.) For $x=(-\alpha, \beta) \in T P_{-}$, similarly set $x_{l}=\alpha$ and $x_{h}=\beta$.

Lemma 2.1. An element $x \in T P$ is uniquely given by $x_{l}, x_{h}$, and the sign of $x$.

Proof. This is obvious.

For convenience, we will often give elements $x \in T P$ in terms of $x_{l}, x_{h}$, and the sign of $x$.

Definition 2.6. For convenience, we will say that $P O^{l}(x)=P O^{l}\left(x_{l}\right)$ and $P O^{r}(x)=P O^{r}\left(x_{l}\right)$ for $T$-pairs $x$.

Definition 2.7. For any pair $x=(\alpha, \beta) \in \bar{\Gamma} \times \bar{\Gamma}$, define $E_{x}$ to be $E_{x}=e_{\alpha} \otimes e_{\beta}$. We will, however, only consider cases where $x=\left(e_{i}-e_{j}, e_{k}-e_{l}\right)$ and $i+k=j+l$.

Lemma 2.2. Suppose $x_{1}, \ldots, x_{n} \in T P$. Then, if $E_{x_{1}} \cdots E_{x_{n}} \neq 0$, then there is a unique $z \in \bar{\Gamma} \times \bar{\Gamma}$ such that $E_{z}=E_{x_{1}} \cdots E_{x_{n}}$. Namely, this is given by componentwise addition of all $x_{i}$.

Proof. This follows from nilpotency. It is easy to see that we need only show that $E_{x_{1}} \cdots E_{x_{n}} \neq e_{i i} \otimes e_{j j}$ for any $i$ and $j$. Equivalently, we have to show that the componentwise addition of all $x_{i}$ is not $(0,0)$.

First, we generalize Ord and $\preceq$. Clearly, every $\alpha \in \tilde{\Gamma}$ can be written as $\alpha=$ $\sum_{i=1}^{m} \alpha_{k_{i}}$ for some $k_{i}$. We say that $\alpha \preceq \beta$ for $\alpha, \beta \in \tilde{\Gamma}$ if $\alpha=\sum_{i=1}^{m} \alpha_{k_{i}}$ and $\beta=$ $\sum_{i=1}^{m} T^{l_{i}} \alpha_{k_{i}}$ for nonnegative integers $l_{i}$. In this case, we define $\widetilde{\operatorname{Ord}}(-\alpha, \beta)=$ $\widetilde{\operatorname{Ord}}(\beta,-\alpha)=\sum_{i} l_{i}$. Note that, when $x \in T P, \widetilde{\operatorname{Ord}}(x)=|x| \operatorname{Ord}(x)$.

Now, we set $\widetilde{T P}_{+}=\{(\beta,-\alpha) \in \tilde{\Gamma} \times-\tilde{\Gamma} \mid \alpha \underline{\Omega} \beta, \alpha \neq \beta\}$ and $\widetilde{T P}_{-}=\{(-\alpha, \beta) \in$ $-\tilde{\Gamma} \times \tilde{\Gamma} \mid \alpha \underline{\preceq} \beta\}$. Let $\widetilde{T P}=\widetilde{T P}-\cup \widetilde{T P}+\cup\{(0,0)\}$ and let $\widetilde{\operatorname{Ord}}(0,0)=0$. We see that componentwise addition of two elements of $\widetilde{T P}$, if in $\bar{\Gamma} \times \bar{\Gamma} \cup\{(0,0)\}$, yields another element of $\widetilde{T P}$, where the orders are summed. In particular, this means that, when $x+y=(0,0)$, for $x, y \in \widetilde{T P}$, then $x=y=(0,0)$. Since $T P \subset \widetilde{T P}$, it follows that any componentwise sum of elements of $T P$, if in $\bar{\Gamma} \times \bar{\Gamma}$, yields a nonzero element of $\widetilde{T P}$. This is all we need.

Definition 2.8. Define $Q: T P^{n} \rightarrow T P \cup\{0\}$ as follows. When $E_{x_{1}} \cdots E_{x_{n}}=$ $E_{z}, z \in \bar{\Gamma} \times \bar{\Gamma}$, for $x_{i} \in T P, Q\left(x_{1}, \ldots, x_{n}\right)=z$. Otherwise, $Q\left(x_{1}, \ldots, x_{n}\right)=0$.

We now apply these general definitions to the task of setting aside the important subsets of $T P \times T P$. These subsets parameterize nonzero terms that arise upon expansion of $\varepsilon$. 
Definition 2.9. Define $C T Q, C T Q_{s}$, and $C T Q_{o}$ (compatible $T$-quadruples of same/opposite sign) by

$$
\begin{gathered}
C T Q=\left\{(x, y) \in T P \times T P \mid E_{x} E_{y} \neq 0\right\}, \\
C T Q_{s}=C T Q \cap\left(T P_{+} \times T P_{+} \cup T P_{-} \times T P_{-}\right), \\
C T Q_{o}=C T Q \cap\left(T P_{+} \times T P_{-} \cup T P_{-} \times T P_{+}\right) .
\end{gathered}
$$

Definition 2.10. Define $C T Q_{s}^{i}, C T Q_{s}^{c}, C T Q_{s}^{d}$ (increasing, constant, and decreasing in order) by

$$
\begin{aligned}
& C T Q_{s}^{i}=\left\{(x, y) \in C T Q_{s} \mid \operatorname{Ord}(x)<\operatorname{Ord}(y)\right\}, \\
& C T Q_{s}^{c}=\left\{(x, y) \in C T Q_{s} \mid \operatorname{Ord}(x)=\operatorname{Ord}(y)\right\}, \\
& C T Q_{s}^{d}=\left\{(x, y) \in C T Q_{s} \mid \operatorname{Ord}(x)>\operatorname{Ord}(y)\right\} .
\end{aligned}
$$

Lemma 2.3. For any $(x, y) \in C T Q_{o},|x| \neq|y|$.

Proof. If $|x|=|y|$, then it is clear from $(x, y) \in C T Q_{o}$ that $x=-y$, negating in each component. But then $\operatorname{Ord}(x)=-\operatorname{Ord}(y)$, which is impossible.

Definition 2.11. Define $C T Q_{o}^{i}$ and $C T Q_{o}^{d}$ (increasing and decreasing in size) by

$$
\begin{aligned}
& C T Q_{o}^{i}=\left\{(x, y) \in C T Q_{o}|| x|<| y \mid\right\}, \\
& C T Q_{o}^{d}=\left\{(x, y) \in C T Q_{o}|| x|>| y \mid\right\} .
\end{aligned}
$$

Note 2.3. $C T Q_{o}$ and $C T Q_{s}$ are partitioned into increasing and decreasing quadruples by different criteria (size and order, respectively)! It turns out that these criteria are the useful ones.

Now we define good and bad subsets of the sets we have defined.

\section{Definition 2.12.}

$$
G C T Q_{s}=\left\{(x, y) \in C T Q_{s} \mid \operatorname{Ord}(x)-\operatorname{Ord}(y) \text { does not divide } \operatorname{Ord}(x)\right\}
$$

and $B C T Q_{*}=C T Q_{*} \backslash G C T Q_{*}$. Moreover, set $G C T Q_{*_{1}}^{*_{2}}=G C T Q_{*_{1}} \cap C T Q_{*_{1}}^{*_{2}}$ and similarly for $B C T Q$.

Note 2.4. The definitions above are symmetric in the two components of $T$ quadruples because $(a-b)$ divides $a$ iff $(a-b)$ divides $b$ iff $(b-a)$ divides $a$ iff $(b-a)$ divides $b$.

Note 2.5. In the following definitions, particularly in the orientation-reversing cases, it may not be completely obvious that the maps have the images indicated (i.e. are well-defined). See Proposition 2.1 for well-definition.

Definition 2.13. We define $\phi: C T Q_{o} \rightarrow C T Q_{s} \cup P T P \cup H P T P_{-}$. We first consider the increasing case. Take $(x, y) \in C T Q_{o}^{i}$ and suppose $x_{l}=e_{j}-e_{j+|x|}$, $x_{h}=e_{k}-e_{k+|x|}, y_{l}=e_{k+|x|-|y|}-e_{k+|x|}$, and $y_{h}=e_{j}-e_{j+|y|}$. Suppose that 
$|x|=p(|y|-|x|)+q$ for $0 \leq q<|y|-|x|$. If $q \neq 0$ (i.e. $(x, y)$ is good), then set $\phi(x, y)=(u, v)$ where $u$ and $v$ have the same sign as $y$ and are given by $u_{l}=e_{k+|x|-|y|+q}-e_{k}, u_{h}=e_{j+|x|}-e_{j+|y|-q}, v_{l}=e_{k+|x|-|y|}-e_{k+|x|-|y|+q}$, and $v_{h}=e_{j+|y|-q}-e_{j+|y|}$. In other words, $u$ and $v$ are the unique same-sign $T$-pairs such that $|v|=q$ and $Q(u, v)=Q(x, y)$. In the case that $(x, y)$ is bad, we simply set $\phi(x, y)=Q(x, y)$.

In the good decreasing case, we take $(y, x) \in G C T Q_{o}^{d}$, set $q$ and $p$ as above, and again let $\phi(y, x)=(v, u)$ where $v, u$ are the unique $T$-pairs with the same sign as $y$ such that $|v|=q$ and $Q(v, u)=Q(y, x)$. In the bad decreasing case, we again set $\phi(y, x)=Q(y, x)$.

Definition 2.14. We define $\psi: C T Q_{s} \backslash C T Q_{s}^{c} \rightarrow C T Q_{o}$ as follows. Take $(u, v) \in C T Q_{s}^{i}$. If $(u, v)$ is $\operatorname{good}$, i.e. $\operatorname{Ord}(v)-\operatorname{Ord}(u)$ does not divide $\operatorname{Ord}(u)$, then write $\operatorname{Ord}(u)=p[\operatorname{Ord}(v)-\operatorname{Ord}(u)]+q$ for $0<q<\operatorname{Ord}(v)-\operatorname{Ord}(u)$. In the case $(u, v)$ is $\operatorname{bad}$, i.e. $\operatorname{Ord}(v)-\operatorname{Ord}(u)$ divides $\operatorname{Ord}(u)$, we again write $\operatorname{Ord}(u)=$ $p[\operatorname{Ord}(v)-\operatorname{Ord}(u)]+q$, this time choosing $q=0$ when $(u, v) \in T P_{-} \times T P_{-}$and $q=\operatorname{Ord}(v)-\operatorname{Ord}(u)$ when $(u, v) \in T P_{+} \times T P_{+}$. Then, we define $\psi(u, v)=(x, y)$ where $y$ has the same sign as $u$ and $v$, while $x$ has the opposite sign, and $x_{l}=\left(T^{q}+T^{q+\operatorname{Ord}(v)-\operatorname{Ord}(u)}+\ldots+T^{q+(p-1)(\operatorname{Ord}(v)-\operatorname{Ord}(u))}\right)\left(u_{l}+v_{l}\right)+T^{\operatorname{Ord}(u)} v_{l}$, $x_{h}=\left(T^{\operatorname{Ord}(v)-\operatorname{Ord}(u)}+T^{2[\operatorname{Ord}(v)-\operatorname{Ord}(u)]}+\ldots+T^{p(\operatorname{Ord}(v)-\operatorname{Ord}(u))}\right)\left(u_{l}+v_{l}\right)+$ $T^{(p+1)(\operatorname{Ord}(v)-\operatorname{Ord}(u))} v_{l}, y_{l}=\left(1+T^{\operatorname{Ord}(v)-\operatorname{Ord}(u)}+\ldots+T^{p(\operatorname{Ord}(v)-\operatorname{Ord}(u))}\right)\left(u_{l}+\right.$ $\left.v_{l}\right)+T^{(p+1)(\operatorname{Ord}(v)-\operatorname{Ord}(u))} v_{l}$, and $y_{h}=\left(T^{q}+T^{q+(\operatorname{Ord}(v)-\operatorname{Ord}(u))}+\ldots+T^{\operatorname{Ord}(u)}\right)$ $\left(u_{l}+v_{l}\right)+T^{\mathrm{Ord}(v)} v_{l}$.

In the case of decreasing quadruples, we begin with $(v, u) \in C T Q_{s}^{d}$ and set $\psi(v, u)=(y, x)$, with $y, x, p$, and $q$ all defined as above.

Definition 2.15. We similarly define $\psi^{r}: P T P^{r} \cup H P T P_{-}^{r} \rightarrow C T Q_{o}^{i}$ and $\psi^{l}: P T P^{l} \cup H P T P_{-}^{l} \rightarrow C T Q_{o}^{d}$. Suppose $v \in P T P^{r} \cup H P T P_{-}^{r}$. If $v$ is good, i.e. $P O^{r}(v)$ does not divide $\operatorname{Ord}(v)$, we write $\operatorname{Ord}(v)=p P O^{r}(v)+q$ for $0<q<$ $P O^{r}(v)$. If $v$ is bad, i.e. $P O^{r}(v)$ divides $\operatorname{Ord}(v)$, we set $q=0$ when $v \in T P_{-}$ and $q=P O^{r}(v)$ when $v \in T P_{+}$, and again write $\operatorname{Ord}(v)=p P O^{r}(v)+q$. Now, we define $\psi^{r}(v)=(x, y)$ where $y$ has the same sign as $v, x$ has the opposite sign, and $x_{l}=\left(T^{q}+T^{q+P O^{r}(v)}+\ldots+T^{q+(p-1) P O^{r}(v)}\right) v_{l}, x_{h}=\left(T^{P O^{r}(v)}+\right.$ $\left.T^{2 P O^{r}(v)}+\ldots+T^{p P O^{r}(v)}\right) v_{l}, y_{l}=\left(1+T^{P O^{r}(v)}+\ldots+T^{p P O^{r}(v)}\right) v_{l}$, and $y_{h}=$ $\left(T^{q}+T^{q+P O^{r}(v)}+\ldots+T^{q+p P O^{r}(v)}\right) v_{l}$. For $v \in P T P^{l} \cup H P T P_{-}^{l}$, we define $\psi^{l}(v)=(y, x)$, with $y, x, p$, and $q$ all defined as above.

Note 2.6. We had to define two separate maps $\psi^{r}$ and $\psi^{l}$ because sometimes $H P T P_{-}^{r} \cup P T P^{r}$ and $H P T P_{-}^{l} \cup P T P^{l}$ intersect, and $\psi^{l}$ and $\psi^{r}$ do not agree.

Definition 2.16. We say that a pair $x=(\beta,-\alpha) \in T P_{+}$reverses orientation if $C_{\alpha, \beta}=1$, and in this case, we set $C_{x}=1$. Otherwise $x$ preserves orientation and $C_{x}=0$. For negative pairs $x=(-\alpha, \beta) \in T P_{-}$, we say $x$ preserves orientation and $C_{x}=1$ if $\alpha=\beta$; otherwise, we set $C_{x}=C_{(\beta,-\alpha)}$ and say that $x$ preserves/reverses orientation iff $(\beta,-\alpha)$ does. 
Definition 2.17. Define $s_{q}, s: T P^{n} \rightarrow \mathbb{Z}\left[q, q^{-1}\right]$ as follows: $s_{q}\left(x_{1}, \ldots, x_{n}\right)=$ $(-q)^{-C_{x_{1}}\left(\left|x_{1}\right|-1\right)-\ldots-C_{x_{n}}\left(\left|x_{n}\right|-1\right)}$ and $s=s_{1}$.

Lemma 2.4. If $(u, v) \in C T Q_{s}^{i}$ then $u$ preserves orientation. In the event $v$ reverses orientation, then $\operatorname{Ord}(u)<\frac{\operatorname{Ord}(v)}{2}$. If $(x, y) \in C T Q_{o}^{i}$, then y preserves orientation. In the event $x$ reverses orientation, $|x| \leq \frac{|y|}{2}$. The same results hold considering $(v, u) \in C T Q_{s}^{d}$ and $(y, x) \in C T Q_{o}^{d}$, respectively.

Proof. In the first case, if $u$ reversed orientation, then $T^{\operatorname{Ord}(u)}\left(v_{l}\right)=v_{h}$, thus $\operatorname{Ord}(u)=\operatorname{Ord}(v)$ by nilpotency - this is a contradiction. On the other hand, when $v$ reverses orientation, $T^{\operatorname{Ord}(v)-\operatorname{Ord}(u)}$ must reverse orientation, and thus by nilpotency cannot be defined on all of $u_{l}+v_{l}$. Thus $\operatorname{Ord}(v)-\operatorname{Ord}(u)<\operatorname{Ord}(u)$, hence the desired result. The second case follows easily from nilpotency.

Proposition 2.1. The maps $\left.\phi\right|_{G C T Q_{o}}: G C T Q_{o} \rightarrow C T Q_{s} \backslash C T Q_{s}^{c}$ and $\psi:$ $C T Q_{s} \backslash C T Q_{s}^{c} \rightarrow G C T Q_{o}$ are inverse to each other. The maps $\left.\phi\right|_{B C T Q_{o}^{i}}$ : $B C T Q_{o}^{i} \rightarrow P T P^{r} \cup H P T P_{-}^{r}$ and $\psi^{r}: P T P^{r} \cup H P T P_{-}^{r} \rightarrow B C T Q_{o}^{i}$ are inverse to each other. Finally, the maps $\left.\phi\right|_{B C T Q_{o}^{d}}: B C T Q_{o}^{d} \rightarrow P T P^{l} \cup H P T P_{-}^{l}$ and $\psi^{l}: P T P^{l} \cup H P T P_{-}^{l} \rightarrow B C T Q_{o}^{d}$ are inverse to each other. All maps $\phi, \psi, \psi^{r}$, and $\psi^{l}$ preserve $Q$ and $s_{q}$.

Proof. The fact that the maps are well-defined, inverse to each other, and preserve $Q$ is easy to see from construction when orientations are preserved (it helps to draw a picture). Also, when orientations are preserved, $s_{q}$ is trivially preserved.

So it remains to consider orientation-reversing cases. Given $(x, y) \in C T Q_{o}^{i}$, if orientation is reversed in $x$ or $y$, it can only be reversed in $x$, and $|x| \leq \frac{|y|}{2}$ by Lemma 2.4. In the case $|x| \neq \frac{|y|}{2}$, it follows that $\phi(x, y)=(u, v) \in C T Q_{s}^{i}$, where $u$ preserves orientation, $v$ reverses orientation, $|v|=|x|$, and $\operatorname{Ord}(y)=$ $\operatorname{Ord}(u)<\operatorname{Ord}(v)-\operatorname{Ord}(u)=\operatorname{Ord}(x)+\operatorname{Ord}(y)$. So $s_{q}$ and $Q$ are preserved, and $\psi(u, v)=(x, y)$. If, instead, $|x|=\frac{|y|}{2}$, then $\phi(x, y)=v$, where $|v|=|x|$, $v \in P T P^{r} \cup H P T P_{-}^{r}$, and $v$ reverses orientation. Again, $s_{q}$ and $Q$ are preserved and $\psi^{r}(v)=(x, y)$.

Now consider an element $(u, v) \in C T Q_{s}^{i} \backslash C T Q_{s}^{c}$ in which either $u$ or $v$ reverses orientation. By Lemma 2.4, only $v$ reverses orientation and $\operatorname{Ord}(u)<\frac{\operatorname{Ord}(v)}{2}$. So, we get $\psi(u, v)=(x, y) \in C T Q_{o}^{i}$ where $\operatorname{Ord}(u)=\operatorname{Ord}(y), \operatorname{Ord}(v)=2 \operatorname{Ord}(y)+$ $\operatorname{Ord}(x),|x|=|v|,|y|=2|v|+|u|$. In this case, $x$ reverses orientation and $y$ does not, so $Q$ and $s_{q}$ are preserved, and clearly $\phi(x, y)=(u, v)$. Finally, suppose $v \in P T P^{r} \cup H P T P_{-}^{r}$ reverses orientation. By definition, this means that $T^{P O^{r}(v)}$ reverses orientation on $v$. By nilpotency, $\operatorname{Ord}(v)<2 P O^{r}(v)$, and it follows that $\psi^{r}(v)=(x, y) \in C T Q_{o}^{i}$. We then have $|x|=|v|, \operatorname{Ord}(v)=2 \operatorname{Ord}(y)+\operatorname{Ord}(x)$, and $P O^{r}(v)=\operatorname{Ord}(x)+\operatorname{Ord}(y)$. It follows that $x$ reverses orientation, and so $s_{q}$ and $Q$ are preserved, and $\phi(x, y)=v$. 
The decreasing and left cases follow in exactly the same way as the increasing and right cases.

Corollary 2.1. Suppose $(x, y) \in C T Q_{o}^{i}$. If $\phi(x, y)=(u, v)$ and not all of $x, y, u, v$ preserve orientation, then $x, v$ reverse orientation, $y, u$ preserve orientation, and $|x|=|v|$. If $\phi(x, y)=v$ and not all of $v, x, y$ preserve orientation, then $x, v$ reverse orientation, $y$ preserves orientation, and $|x|=|v|$. The same facts hold under the assumptions $(y, x) \in C T Q_{o}^{d}$ with $\phi(y, x)=(v, u)$ or $v$.

Proof. This follows directly from the argument above.

Lemma 2.5. $\sum_{(x, y) \in C T Q_{s}^{c}} s(x, y) E_{Q(x, y)}=\sum_{x \in T P}(1-|x|) s(x) C_{x} E_{x}$.

Proof. Fix a choice of sign \pm for this proof. Clearly, whenever $E_{x} E_{y} \neq 0$, $\operatorname{Ord}(x)=\operatorname{Ord}(y)$, and $x, y \in T P_{ \pm}$, then $C_{x}=C_{y}=1$. In this case, $s(x) s(y)=$ $-s(Q(x, y))$, as $Q(x, y)$ also has reversed orientation and the same order as $x$ and $y$. It remains only to see that, for any $z \in T P_{ \pm}$with $C_{z}=1$, there are $|z|-1$ ways of writing $E_{z}=E_{x} E_{y}$ for $x, y \in T P_{ \pm}$, and they all are of this form. The formula follows immediately.

Direct proof of Proposition 1.3. Set $b=\sum_{i} e_{i i} \otimes e_{i i}$ and $P_{-}=\sum_{i<j} e_{j i} \otimes e_{i j}$, so that $r_{s t}=b+P_{-}$. Then, using $\phi$ and $\psi$,

$$
a^{2}+a r_{s t}+r_{s t} a=a^{2}+a P_{-}+P_{-} a+\frac{1}{2}(a b+b a)
$$

$$
\begin{gathered}
=\sum_{(x, y) \in C T Q_{s}} s(x, y) E_{Q(x, y)}-\sum_{(x, y) \in C T Q_{o}} s(x, y) E_{Q(x, y)}-\frac{1}{2} \sum_{x \in H P T P} S_{x} s(x) E_{x} \\
=-\sum_{x \in H P T P_{-} \cup P T P} s(x) E_{x}+\sum_{(x, y) \in C T Q_{s}^{c}} s(x, y) E_{Q(x, y)}-\frac{1}{2} \sum_{x \in H P T P} S_{x} s(x) E_{x} \\
=\sum_{x \in T P}(1-|x|) s(x) C_{x} E_{x}-\sum_{x \in P T P} s(x) E_{x}-\frac{1}{2} \sum_{x \in H P T P} s(x) E_{x} .
\end{gathered}
$$

Equation (1.8) follows immediately.

Remark 2.1. The combinatorial formula 1.8 for $\varepsilon$ also follows from the proof of Theorem 1.2. Namely, in the proof we actually show that $R_{J}$ has the form of $R_{\mathrm{GGS}}$ but we use the combinatorial formula (1.8) for $\varepsilon$ instead of the original one. On the other hand, since the combinatorial formula (1.8) is symmetric, and because $R_{J}$ satisfies the QYBE and the Hecke relation, $R_{J}$ must be the unique element satisfying the hypotheses of Proposition 1.1 by the discussion in Section 1.2. The proof of Proposition 1.3 above is, however, given for pedagogical reasons and because the results used will be needed later. 


\subsection{Passing properties of $T$-quadruples.}

Overview 2.2. By passing properties of a $T$-pair we mean information about its left- and right-passing order. In particular, usually we will be concerned with whether a pair is (half) right- or (half) left-passed.

In this section, we will list all possible passing properties of compatible increasing and decreasing quadruples, in connection with those properties of their images under $\phi$ or $\psi$. These results, Lemmas 2.7 and 2.8, are essential in order to consider quadratic terms which arise in the Hecke condition for $R_{\mathrm{GGS}}$, which are similar to those in the formula for $\varepsilon$ but include powers of $q$ which depend on the passing properties. As a consequence of these results, one can prove the Hecke condition for $R_{\mathrm{GGS}}$ directly (see [S2]).

In order to prove these results, we will first need to develop some more powerful combinatorial tools and notation regarding Belavin-Drinfeld triples. The combinatorics can best be pictured on the Dynkin diagram for $\mathfrak{s l}(n)$. We picture this diagram as the line segment $[1, n]$ with integer vertices. We then picture the positive root $e_{i}-e_{j}$, for $1 \leq i<j \leq n$, as the line segment $[i, j]$. In this context, $\Gamma_{1}$ and $\Gamma_{2}$ can be thought of as subsets of the graph consisting of the union of all the length-1 segments which make them up, and maps $T$ are nilpotent graph isomorphisms $\Gamma_{1} \rightarrow \Gamma_{2}$.

Definition 2.18. Assume $i<j$ and $k<l$. We say $e_{i}-e_{j}<e_{k}-e_{l}$ if $i<k$ $\left(e_{i}-e_{j}\right.$ is to the left of $\left.e_{k}-e_{l}\right)$. As subcases of this, we say that $e_{i}-e_{j} \ll e_{k}-e_{l}$ for $j<k, e_{i}-e_{j} \lessdot e_{k}-e_{l}$ if $j=k$, and $e_{i}-e_{j}<e_{k}-e_{l}$ for $j>k$. Similarly define $>$ by $\alpha>\beta$ whenever $\beta<\alpha$, and the same for $\gg$, $\gg$, and $>$. If $\alpha \ll \beta$ or $\beta \ll \alpha$, then we say that $\alpha \perp \beta$ (meaning there are orthogonal subsets $X, Y \subset \Gamma$ so that $\alpha \in \tilde{X}$ and $\beta \in \tilde{Y})$.

Here and in the sequel we will make frequent use of the following key combinatorial lemma:

Definition 2.19. Take $\alpha \in \tilde{\Gamma}_{1}$. Let $M_{\alpha}$ be the smallest positive integer such that $T^{M_{\alpha}-1} \alpha \in \tilde{\Gamma}_{2} \backslash \tilde{\Gamma}_{1}$. Let $c_{\alpha}$ be the smallest positive integer less than $M_{\alpha}$ such that $T^{c_{\alpha}} \alpha \not \perp \alpha$, if such an integer exists. Otherwise set $c_{\alpha}=\infty$. If $c_{\alpha}$ is finite and there is a positive integer $d<M_{\alpha}$ which is not a multiple of $c_{\alpha}$ satisfying $T^{d} \alpha \not \perp \alpha$, then let $d_{\alpha}$ be the smallest such $d$. Otherwise set $d_{\alpha}=\infty$.

Lemma 2.6. Suppose $\alpha \in \tilde{\Gamma}_{1}$ and $c_{\alpha}, d_{\alpha}<\infty$. (i) For any positive integer $d<M_{\alpha}$ such that $T^{d} \alpha \not \perp \alpha$, either $d$ is a multiple of $c_{\alpha}$, or $d=d_{\alpha}$. (ii) $d_{\alpha}+c_{\alpha}-\operatorname{gcd}\left(c_{\alpha}, d_{\alpha}\right) \geq M_{\alpha}$ and $T^{d_{\alpha}} \alpha \perp T^{c_{\alpha}} \alpha$.

Proof. Take some $d$ such that $T^{d} \alpha \not \perp \alpha$. We show in the following paragraph that either $c_{\alpha}$ divides $d$, or $c_{\alpha}+d-\operatorname{gcd}\left(d, c_{\alpha}\right) \geq M_{\alpha}$ and $T^{d} \alpha \perp T^{c_{\alpha}} \alpha$. This proves the lemma - all that remains is to see that, in the latter case, $d=d_{\alpha}$. If, instead, $d \neq d_{\alpha}$, then applying the above result also to $d_{\alpha}$, we find that both $T^{d} \alpha$ and $T^{d_{\alpha}} \alpha$ are perpendicular to $T^{c_{\alpha}} \alpha$. By space concerns on the diagram, 
it follows that $T^{d} \alpha \not \perp T^{d_{\alpha}} \alpha$, but then $T^{d-d_{\alpha}} \alpha \not \perp \alpha$, which would show that $c_{\alpha}$ divides $d-d_{\alpha}$, in contradiction to $d_{\alpha}+c_{\alpha}-\operatorname{gcd}\left(c_{\alpha}, d_{\alpha}\right) \geq M_{\alpha}$.

So, take any $d$ such that $T^{d} \alpha \not \perp \alpha$, and assume that $d$ is not a multiple of $c_{\alpha}$. Define $f:\left\{0, \ldots, M_{\alpha}-1\right\} \times \Gamma_{1} \rightarrow\{-1,1\}$ by $f\left(p, \alpha_{i}\right)=1$ if $T^{p}$ preserves orientation on $\alpha_{i}$, and $f\left(p, \alpha_{i}\right)=-1$ otherwise. Define $g:\left\{0, \ldots, M_{\alpha}-1\right\} \times \Gamma_{1} \rightarrow$ $\mathbb{Z}$ as follows. For any $1 \leq i \leq n-1$ ( $n$ is the length of the Dynkin diagram), let $q$ be given by $T^{p} \alpha_{i}=\alpha_{q}$. Then we define $g\left(p, \alpha_{i}\right)=q-f\left(\alpha_{i}\right) i$. Define $F=f \times g$. Clearly, $F$ is defined so that if $Y \subset \Gamma_{1}$ is a connected segment of the diagram, then $F$ is constant on $\{p\} \times Y$, for each fixed $p, 0 \leq p \leq$ $M_{\alpha}-1$. Since $T^{a c_{\alpha}} \alpha \not \perp T^{(a-1) c_{\alpha}} \alpha$ for $a c_{\alpha}<M_{\alpha}$, it follows that $F$ is $c_{\alpha^{-}}$ periodic in the first component. For the same reason, $F$ is $d$-periodic in the first component. If $d+c_{\alpha}-\operatorname{gcd}\left(d, c_{\alpha}\right)<M_{\alpha}, F$ must be $\operatorname{gcd}\left(d, c_{\alpha}\right)$-periodic in the first component. This follows since $F\left(d+l, \alpha_{i}\right)=F\left(l, \alpha_{i}\right), 0 \leq l<c_{\alpha}-\operatorname{gcd}\left(d, c_{\alpha}\right)$ implies $F\left(a, \alpha_{i}\right)=F\left(b, \alpha_{i}\right)$ whenever $a \equiv b\left(\bmod \operatorname{gcd}\left(d, c_{\alpha}\right)\right), 0 \leq a, b<M_{\alpha}$. By minimality, $\operatorname{gcd}\left(d, c_{\alpha}\right)=c_{\alpha}$, which is impossible. Hence, $d+c_{\alpha}-\operatorname{gcd}\left(d, c_{\alpha}\right) \geq$ $M_{\alpha}$. Furthermore, $T^{d} \perp T^{c_{\alpha}}$, because otherwise $T^{d-c_{\alpha}} \alpha \not \perp \alpha$, which would imply that $d-\operatorname{gcd}\left(d, c_{\alpha}\right) \geq M_{\alpha}$ by the above results applied to $d-c_{\alpha}$, which is clearly contradictory.

Corollary 2.2. Suppose that $T^{l}\left(\alpha_{i}\right)=\alpha_{i+r}$ for $a \leq i<b$ where $r \leq b-a$ and $l>0$. Then for any $\alpha=e_{c}-e_{c+s}, \beta=e_{d}-e_{d+s}$, where $s \geq r$ and $a \leq c<d \leq b$, then $\beta \nprec \alpha$, and $\alpha \prec \beta$ implies that $\operatorname{Ord}(\beta,-\alpha)=\frac{l(d-c)}{r}$.

By reversing all directions, given $T^{l}\left(\alpha_{i}\right)=\alpha_{i-r}, a \leq i<b$, with $r \leq b-a$, then for any $\alpha=e_{c-s}-e_{c}, \beta=e_{d-s}-e_{d}$, where $s \geq r$ and $a \leq d<c \leq b$, then $\beta \nprec \alpha$, and $\alpha \prec \beta$ implies $\operatorname{Ord}(\beta,-\alpha)=\frac{l(c-d)}{r}$.

Proof. First, it is clear that $r$ divides $c-d$ iff $T^{k l} \alpha=\beta$ for $k>0$ an integer. In this case, the theorem is satisfied; so suppose not. By applying $T^{ \pm l}$ some number of times to $\alpha$ or $\beta$, it suffices to assume $0<d-c<r$. Now, if $T^{l}$ is defined on $\alpha$, then $\alpha<\beta<T^{l} \alpha$ together with the Lemma gives the desired result. So assume $T^{l}$ is not defined on $\alpha$, and hence it is not defined on $\beta$ either. Suppose $T^{m} \beta=\alpha$ for some positive integer $m$. Write $\beta=e_{i}-e_{j}$. Now, by applying $T^{m}$ some number of times to each $\alpha_{p}, i \leq p<j$, we can obtain $\alpha_{q}$ for some $a \leq q \leq b$, showing that $T^{l}$ is defined on $\beta$, which is a contradiction. But then $T^{m}$ is defined The direction-reversed case is the same.

Definition 2.20. For convenience, let $E P T P^{*}=H P T P^{*} \cup P T P^{*}$ for any or no superscript $*$.

Definition 2.21. When $T^{k}$ acts on some segment of the Dynkin diagram (i.e. some subset of $\Gamma$ ) by sending $\alpha_{i}$ to $\alpha_{i+k}$, we say it acts by shifting to the right by $k$ when $k$ is positive, and by shifting to the left by $k$ when $k$ is negative. In particular, on each segment, $T^{k}$ acts by shifting iff orientation is preserved.

The next two lemmas summarize all of the possible passing properties of an opposite-sign quadruple and its image under $\phi$. 
Lemma 2.7. Take $(x, y) \in G C T Q_{o}^{i}$ and $\phi(x, y)=(u, v)$. Then exactly one of the following must hold:

(a) $P^{l}(v)=P^{r}(y)+P^{l}(x), P^{l}(y)=0$

(b) $P^{l}(v)=P^{l}(y), P^{l}(x)=P^{r}(y)=0$

Similarly, exactly one of the following must hold:

(c) $P^{r}(v)=P^{r}(x)+P^{r}(u), P^{l}(u)=0$

(d) $P^{r}(v)=P^{l}(u), P^{r}(u)=P^{r}(x)=0$.

These results, after interchanging superscripts of $l$ with $r$, also hold when one considers $(y, x) \in G C T Q_{o}^{d}$ and $\phi(y, x)=(v, u)$, instead of the original hypothesis.

Proof. Let $\operatorname{Ord}(v)=(k+1) \operatorname{Ord}(y)+k \operatorname{Ord}(x)$ and $\operatorname{Ord}(u)=k \operatorname{Ord}(y)+$ $(k-1) \operatorname{Ord}(x)$ for some $k$ (which exists by construction).

Suppose that $v \in E P T P^{l}$. We will analyze all possible cases by considering the value of $P O^{l}(v)$. Write $P O^{l}(v)=p[\operatorname{Ord}(x)+\operatorname{Ord}(y)]+q$ where $q<\operatorname{Ord}(x)+\operatorname{Ord}(y)$. First, I claim that $p \geq k-1$. Suppose instead that $p<k-1$. In this case, $k \geq 2$, which immediately implies from the proof of Proposition 2.1 that $T^{\operatorname{Ord}(x)+\operatorname{Ord}(y)}$ preserves orientation on $u_{l}+v_{l}$. So $v$ preserves orientation, and by definition $T^{P O^{l}(v)}$ preserves orientation on $v$. Now, if we set $\omega=T^{-\operatorname{Ord}(y)} x_{l}$, we find that $T^{q} \omega \overline{<} \omega$ because $p<k$. Now, $k \geq 2$ shows that $T^{\operatorname{Ord}(x)+\operatorname{Ord}(y)} \omega>\omega$. Finally, $T^{\operatorname{Ord}(x)+\operatorname{Ord}(y)} \omega>T^{q} \omega$, because $p<k-1$. These facts, however, contradict Lemma 2.6.

So, it must be that $p \geq k-1$. We divide into the two cases, (1) $p=k-1$ and $(2) p=k$.

First consider the case $k=p-1$. Set $t=k[\operatorname{Ord}(x)+\operatorname{Ord}(y)]-P O^{l}(v)$. Then we have three cases: (i) $t<\operatorname{Ord}(y)$, (ii) $t=\operatorname{Ord}(y)$, and (iii) $t>\operatorname{Ord}(y)$. First consider (i). Now, $T^{t}\left(y_{l}\right)>y_{l}$, so $y \in P T P^{r}$. Conversely, whenever $y \in P T P^{r}$, clearly $v \in P T P^{l}$ with $P O^{l}(v)=k[\operatorname{Ord}(x)+\operatorname{Ord}(y)]-P O^{r}(y)$ (we use that $y$ always preserves orientation). In this case, $t<\operatorname{Ord}(y)$ and $p=k-1$, as desired. This situation, characterized by $v \in P T P^{l}, y \in P T P^{r}$, falls into (a) and we will call it (a1).

Next, take (ii). In this case, $P O^{l}(v)=k \operatorname{Ord}(x)+(k-1) \operatorname{Ord}(y)$, so $x \in$ $H P T P^{l}$ and $y \in H P T P^{r}$. Conversely, it is clear that $y \in H P T P^{r}$ iff $x \in$ $H P T P^{l}$ from the construction of $\phi$, and in this case, $v \in P T P^{l}$ with $P^{l}(v)=$ $k \operatorname{Ord}(x)+(k-1) \operatorname{Ord}(y)$. Thus, $t=\operatorname{Ord}(y)$ and $p=k-1$, as desired. This is a different case of (a), so let us call it (a2).

Finally, consider (iii). In this case, $0<\operatorname{Ord}(x)+\operatorname{Ord}(y)-t<\operatorname{Ord}(x)$. Set $t^{\prime}=\operatorname{Ord}(x)+\operatorname{Ord}(y)-t=P O^{l}(v)-(k-1)[\operatorname{Ord}(x)+\operatorname{Ord}(y)]$. Then it follows that $T^{t^{\prime}}\left(x_{l}\right) \lessdot x_{l}$, so that $x \in P T P^{l}$ with $P O^{l}(x)=t^{\prime}$. Conversely, if $x \in P T P^{l}$, then $v \in P T P^{l}$ with $P O^{l}(v)=(k-1)[\operatorname{Ord}(x)+\operatorname{Ord}(y)]+P O^{l}(x)$, as desired (this can be checked separately when $x$ reverses orientation-here $k=1$ so there is no difficulty.) Hence, $t>\operatorname{Ord}(y)$ and $p=k-1$. This is the final case of (a), so let us call it (a3).

Next, consider the case $k=p$. Set $t=P O^{l}(v)-k[\operatorname{Ord}(x)+\operatorname{Ord}(y)]$. Because $k=p$, it follows that $0<t \leq \operatorname{Ord}(y)$, hence $y \in E P T P^{l}$ with $\operatorname{PO}^{l}(y)=t$. 
Since $P O^{l}(y)=\operatorname{Ord}(y)$ iff $P O^{l}(v)=\operatorname{Ord}(v)=(k+1) \operatorname{Ord}(y)+k \operatorname{Ord}(x)$, we have $P^{l}(v)=P^{l}(y)$. Conversely, whenever $y \in E P T P^{l}$, then $v \in E P T P^{l}$ with $P O^{l}(v)=P O^{l}(y)+k[\operatorname{Ord}(x)+\operatorname{Ord}(y)]$. Hence, $p=k$ and $P^{l}(v)=P^{l}(y)$. This accounts for case $(\mathrm{b})$.

We have proved the first part of the Lemma, because we have considered all possible nonzero values of $P^{l}(v), P^{l}(x), P^{l}(y)$, and $P^{r}(y)$, and grouped them into the cases (a1), (a2), (a3), and (b). We have shown that each of these is associated with different values of $P O^{l}(v)$, which justifies the zero values of $P^{l}(v), P^{l}(x), P^{l}(y)$, and $P^{r}(y)$ in each case.

Next, we apply the same analysis used in the first part to show that exactly one of (c),(d) holds. Let $t=\left|P O^{r}(v)-\operatorname{Ord}(x)-\operatorname{Ord}(y)\right|$ and $\omega=$ $v_{l}+u_{l}+T^{\operatorname{Ord}(x)+\operatorname{Ord}(y)} v_{l}$. We suppose that $v \in E P T P^{r}$ and divide into the cases $P O^{r}(v)>\operatorname{Ord}(x)+\operatorname{Ord}(y)$ and $P O^{r}(v)<\operatorname{Ord}(x)+\operatorname{Ord}(y)$.

First consider $P O^{r}(v)>\operatorname{Ord}(x)+\operatorname{Ord}(y)$. Then it is clear that $t \leq \operatorname{Ord}(u)$, with equality iff $v \in H P T P^{r}$. Now, $T^{t}$ is defined on $\omega$, and $T^{t} \omega \not \perp \omega$, so $T^{t}$ preserves orientation on $\omega$. This shows that $T^{t} u_{l} \lessdot u_{l}$, so that $u \in E P T P^{l}$ with $P O^{l}(u)=t$. That is, $P^{l}(u)=P^{r}(v)$. Conversely, whenever $u \in E P T P^{l}$, we know from the fact that $u$ preserves orientation that $T^{P O^{l}(u)}$ preserves orientation on $u_{l}$, and hence that $P^{r}(v)=P^{l}(u)$ with $P O^{r}(v)=P O^{l}(u)+\operatorname{Ord}(x)+$ $\operatorname{Ord}(y)$. It is then clear that $P O^{r}(v)>\operatorname{Ord}(x)+\operatorname{Ord}(y)$. This accounts for case (d).

Now, suppose that $v \in E P T P^{r}$ with $P O^{r}(v)<\operatorname{Ord}(x)+\operatorname{Ord}(y)$. We divide into the cases $k \geq 2$ and $k=1$.

First suppose $k \geq 2$. In particular, this implies that $T^{P O^{r}(v)}$ is defined on $\omega$, and by nilpotency, it must preserve orientation. Also, $T^{\operatorname{Ord}(x)+\operatorname{Ord}(y)}$ must preserve orientation on $\omega$. So, we see that $T^{t} u_{l} \gg u_{l}$, thus $u_{l} \in P T P^{r}$ with $P O^{r}\left(u_{l}\right)=t$. Conversely, if $k \geq 2$ and $u \in E P T P^{r}$, we see from $\omega<T^{P O^{r}(u)} \omega<T^{\operatorname{Ord}(x)+\operatorname{Ord}(y)} \omega$ and Lemma 2.6 that $P O^{r}(u)<\operatorname{Ord}(x)+\operatorname{Ord}(y)$. It follows that $u, v \in P T P^{r}$ with $P O^{r}(v)=t$, and $P O^{r}(v)<\operatorname{Ord}(x)+\operatorname{Ord}(y)$, as desired. This accounts for one situation of (c); call this (c1).

Next, suppose $k=1$ and $P O^{r}(v)<\operatorname{Ord}(x)+\operatorname{Ord}(y)$. We further divide into the three cases (i) $P O^{r}(v)<\operatorname{Ord}(x)$, (ii) $P O^{r}(v)=\operatorname{Ord}(x)$, and (iii) $P O^{r}(v)>\operatorname{Ord}(x)$.

In case (i), we use that $y$ preserves orientation to see that $T^{P O^{r}(v)}\left(x_{l}\right)>$ $x_{l}$, so that $x \in P T P^{r}$ with $P O^{r}(x)=P O^{r}(v)$. Conversely, whenever $x \in$ $P T P^{r}$, it follows that $k=1$ using Lemma 2.6: otherwise we would have

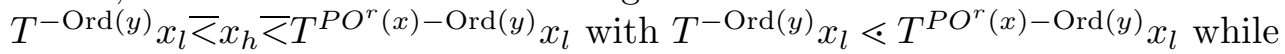
$-\operatorname{Ord}(y)<P O^{r}(x)-\operatorname{Ord}(y)<\operatorname{Ord}(x)$. Hence, $v \in P T P^{r}$ with $P O^{r}(v)=$ $P O^{r}(x)<\operatorname{Ord}(x)$. Call this situation (c3).

In case (ii), we have that $x, u \in H P T P^{r}$. Conversely, whenever $x \in H P T P^{r}$, we must have $k=1$ by Lemma 2.6, and then $u \in H P T P^{r}$ and $P O^{r}(v)=$ $P O^{r}(x)$, as desired. If $u \in H P T P^{r}$, then it also follows from Lemma 2.6 considering $\omega$ that $k=1$, and then $x \in H P T P^{r}$. Call this situation (c2). 
Finally, we consider case (iii). Now, $P O^{r}(v)>\operatorname{Ord}(x)$ shows that $\operatorname{Ord}(x)+$ $\operatorname{Ord}(y)-P O^{r}(v)<\operatorname{Ord}(y)$. By nilpotency, $T^{t}$ must preserve orientation on $y_{l}$, and it follows that $u \in P T P^{r}$ with $P O^{r}(u)=t$. Conversely, if $u \in P T P^{r}$ with $k=1$, then it follows that $P O^{r}(u)<\operatorname{Ord}(u)=\operatorname{Ord}(y)$, so $T^{\operatorname{Ord}(x)+\operatorname{Ord}(y)-P O^{r}(u)} v_{l}>v_{l}$. Hence, $v \in P T P^{r}$ with $P O^{r}(v)=\operatorname{Ord}(x)+$ $\operatorname{Ord}(y)-P O^{r}(u)$, and $\operatorname{Ord}(x)<P O^{r}(v)<\operatorname{Ord}(x)+\operatorname{Ord}(y)$. This has the same passing properties as (c1), so call this situation (c1').

We have finished the second half of the Lemma, since we have accounted for all possible values of $P^{r}(u), P^{l}(u), P^{r}(v)$, and $P^{r}(x)$ in cases (d), (c1), (c1'), $(\mathrm{c} 2)$, and (c3). Each of these are associated with distinct values of $P^{r}(v)$ with rexspect to the $\operatorname{Ord}(x), \operatorname{Ord}(y), \operatorname{Ord}(u)$, and $\operatorname{Ord}(v)$, once $k$ is fixed.

To obtain the result for decreasing quadruples, simply reverse all directions and permute the components of all $T$-quadruples (not pairs!) in this proof.

Lemma 2.8. Take $(x, y) \in B C T Q_{o}^{i}$ and $\phi(x, y)=v$. Then $v \in E P T P^{r}$, $P^{r}(v)+P^{r}(x)=1$, and exactly one of the following hold:

(a) $P^{l}(v)=P^{r}(y)+P^{l}(x), P^{l}(y)=0$.

(b) $P^{l}(v)=P^{l}(y), P^{l}(x)=P^{r}(y)=0$.

Under the hypotheses $(y, x) \in B C T Q_{o}^{d}$ and $\phi(y, x)=v$, these results still hold upon interchanging superscripts of $l$ and $r$.

Proof. Clearly $v \in H P T P^{r}$ iff $x \in H P T P^{r}$. Suppose $v \in P T P^{r}$. Hence $\operatorname{Ord}(x) \in\left\{P O^{r}(v), 0\right\}$. Suppose for a contradiction that $x \in P T P^{r}$. Then, $P O^{r}(x)<P O^{r}(v)$. However, $x<T^{P O^{r}(v)} x \overline{<} T^{P O^{r}(x)} x, x \lessdot T^{P O^{r}(x)} x$ and Lemma 2.6 imply that $P O^{r}(x)=\frac{P O^{r}(v)|x|}{|v|}>P O^{r}(v)$, a contradiction. So, the identity $P^{r}(v)+P^{r}(x)=1$ easily follows (since $v \in E P T P^{r}$ ).

The rest of the proof is almost exactly the same as the proof of the first part of Lemma 2.7, getting rid of $u$. Again, the results follow with simple modifications in the decreasing case.

\subsection{Repeated application of $\phi$ on larger collections of $T$-pairs.}

Overview 2.3. In this section, we consider the longer monomial terms that arise in the expansion of the formula for $R_{J}$. As in the previous cases, most terms cancel; we therefore explicitly give the groupings which cancel (Corollary 2.4 ) or almost cancel, and show that what remains is simply $R_{\mathrm{GGS}}$, proving Theorem 1.2. In order to do this, we need to define the notion of a $T$-chain, which generalizes $T$-quadruples to the objects needed to handle the longer monomials, and prove some more combinatorial results regarding these.

Definition 2.22. A $n, m T P$-chain is a chain $x=\left(x_{1}, \ldots, x_{n}\right) \in T P_{+}^{m} \times$ $T P_{-}^{n-m}$, so that $E_{x_{1}} \cdots E_{x_{n}} \neq 0$. Let $T P C_{n, m}$ denote the set of $n, m$ TP-chains. Let $T P C$ denote the set of all $T P$-chains, i.e. $T P C=\cup_{i>j} T P C_{i, j}$.

Definition 2.23. A chain $\left(x_{1}, \ldots, x_{n}\right) \in T P C_{n, m}$ is said to be outer if $\operatorname{Ord}\left(x_{1}\right)>\ldots>\operatorname{Ord}\left(x_{m}\right)$ and $\operatorname{Ord}\left(x_{m+1}\right)<\ldots<\operatorname{Ord}\left(x_{n}\right)$. Let $T P C_{n, m}^{o}$ 
denote the set of such $n, m$-chains, and let $T P C^{o}$ denote the set of all outer chains for any $n, m$.

Note 2.7. Lemmas 2.9-2.13 below have obvious analogues obtained by changing the sign of all $T$-pairs, reversing the order of $T$-chains, and replacing $T P C_{n, m}$ with $T P C_{n, n-m}$ in all forms. These results are not stated but will be referred to in the same manner as the actual results stated.

Lemma 2.9. ( $i)$ Suppose $\left(x_{1}, x_{2}, x_{3}\right) \in T P C_{3,3}$. Suppose $\psi\left(x_{2}, x_{3}\right)=\left(y_{2}, y_{3}\right)$ and $\psi\left(x_{1}, y_{2}\right)=\left(z_{1}, z_{2}\right)$ where $\left(x_{2}, x_{3}\right),\left(x_{1}, y_{2}\right) \in C T Q_{s}^{d}$. Then $\operatorname{Ord}\left(x_{1}\right)<$ $\operatorname{Ord}\left(x_{2}\right)$ iff $\operatorname{Ord}\left(z_{2}\right)<\operatorname{Ord}\left(y_{3}\right), \operatorname{Ord}\left(x_{1}\right)=\operatorname{Ord}\left(x_{2}\right)$ iff $\operatorname{Ord}\left(z_{2}\right)=\operatorname{Ord}\left(y_{3}\right)$, and $\operatorname{Ord}\left(x_{1}\right)>\operatorname{Ord}\left(x_{2}\right)$ iff $\operatorname{Ord}\left(z_{2}\right)>\operatorname{Ord}\left(y_{3}\right)$.

(ii) Suppose $\left(x_{1}, x_{2}\right) \in T P C_{2,2}$. Suppose $\psi^{l}\left(x_{2}\right)=\left(y_{2}, y_{3}\right)$ and $\psi\left(x_{1}, y_{2}\right)=$ $\left(z_{1}, z_{2}\right)$ where $x_{2} \in P T P^{l}$ and $\left(x_{1}, y_{2}\right) \in C T Q_{s}^{d}$. Then $\operatorname{Ord}\left(x_{1}\right)$ ? Ord $\left(x_{2}\right)$ iff $\operatorname{Ord}\left(z_{2}\right)$ ? Ord $\left(y_{3}\right)$ for ? any order relation $=,<$, or $>$.

Proof. The proof is the same for both (i) and (ii), does not mention $x_{3}$, and is given in the following paragraphs. First, we note that $\operatorname{Ord}\left(x_{1}\right)=\operatorname{Ord}\left(x_{2}\right)$ iff $x_{1}$ and $x_{2}$ reverse orientation, which is true iff $z_{2}$ and $y_{3}$ reverse orientation, which is true iff $\operatorname{Ord}\left(z_{2}\right)=\operatorname{Ord}\left(y_{3}\right)$.

Now, we show that $\operatorname{Ord}\left(x_{1}\right)<\operatorname{Ord}\left(x_{2}\right)$ implies $\operatorname{Ord}\left(z_{2}\right)<\operatorname{Ord}\left(y_{3}\right)$. To reach a contradiction, suppose that $\operatorname{Ord}\left(x_{1}\right)<\operatorname{Ord}\left(x_{2}\right)$ and $\operatorname{Ord}\left(z_{2}\right)>\operatorname{Ord}\left(y_{3}\right) . \operatorname{By}$ nilpotency, $x_{1}$ and $y_{3}$ preserve orientation. Thus $z_{2}$ and $x_{2}$ also preserve orientation. Write $\operatorname{Ord}\left(y_{2}\right)=p \operatorname{Ord}\left(z_{1}\right)+(p-1) \operatorname{Ord}\left(z_{2}\right)$.

First suppose $p \geq 2$. Then $\left|z_{2}\right|>\frac{1}{2}\left|z_{1}\right|$, so that $T^{\operatorname{Ord}\left(z_{1}\right)+\operatorname{Ord}\left(z_{2}\right)}$ is defined on $\left(y_{3}\right)_{h}$ and therefore on $\left(y_{3}\right)_{l}$. However, this implies that $T^{\operatorname{Ord}\left(y_{3}\right)+\operatorname{Ord}\left(z_{1}\right)}\left(\left(z_{2}\right)_{l}+\right.$ $\left.\left(y_{3}\right)_{l}\right)>\left(z_{2}\right)_{l}+\left(y_{3}\right)_{l}>T^{\operatorname{Ord}\left(z_{1}\right)+\operatorname{Ord}\left(z_{2}\right)}\left(\left(z_{2}\right)_{l}+\left(y_{3}\right)_{l}\right)$, while $T^{\operatorname{Ord}\left(y_{3}\right)+\operatorname{Ord}\left(z_{1}\right)}\left(\left(z_{2}\right)_{l}+\right.$ $\left.\left(y_{3}\right)_{l}\right)>T^{\operatorname{Ord}\left(z_{1}\right)+\operatorname{Ord}\left(z_{2}\right)}\left(\left(z_{2}\right)_{l}+\left(y_{3}\right)_{l}\right)$. This contradicts Lemma 2.6.

So $p=1$. Then $\operatorname{Ord}\left(x_{1}\right)=2 \operatorname{Ord}\left(z_{1}\right)+\operatorname{Ord}\left(z_{2}\right)$ and $\operatorname{Ord}\left(y_{2}\right)=\operatorname{Ord}\left(z_{1}\right)$. Since $\operatorname{Ord}\left(x_{1}\right)<\operatorname{Ord}\left(x_{2}\right)$, it must be that $\operatorname{Ord}\left(x_{2}\right)>2 \operatorname{Ord}\left(z_{1}\right)+\operatorname{Ord}\left(y_{3}\right)=$ $2 \operatorname{Ord}\left(y_{2}\right)+\operatorname{Ord}\left(y_{3}\right)$, which implies that $\left|y_{3}\right|>\frac{1}{2}\left|y_{2}\right|$. So $T^{\operatorname{Ord}\left(y_{2}\right)+\operatorname{Ord}\left(y_{3}\right)}\left[\left(z_{2}\right)_{l}+\right.$ $\left.\left(y_{3}\right)_{l}\right]<\left(z_{2}\right)_{l}+\left(y_{3}\right)_{l}$. Now, write $\operatorname{Ord}\left(z_{1}\right)+\operatorname{Ord}\left(z_{2}\right)=q\left[\operatorname{Ord}\left(y_{2}\right)+\operatorname{Ord}\left(y_{3}\right)\right]+m$, for $0 \leq m<\operatorname{Ord}\left(y_{2}\right)+\operatorname{Ord}\left(y_{3}\right)$. Since $\operatorname{Ord}\left(y_{2}\right)+\operatorname{Ord}\left(y_{3}\right)<\operatorname{Ord}\left(z_{1}\right)+\operatorname{Ord}\left(z_{2}\right)<$ $\operatorname{Ord}\left(x_{2}\right)-\operatorname{Ord}\left(y_{2}\right)$, it follows that $T^{m}\left(\left(y_{3}\right)_{l}+\left(z_{2}\right)_{l}\right) \lessdot\left(y_{3}\right)_{l}+\left(z_{2}\right)_{l}(q=1)$ or $T^{m}\left(\left(y_{3}\right)_{l}+\left(z_{2}\right)_{l}\right) \overline{<}\left(y_{3}\right)_{l}+\left(z_{2}\right)_{l}(q>1)$. By Lemma 2.6, it follows that $c_{\left(y_{3}\right)_{l}+\left(z_{2}\right)_{l}}$ divides both $m$ and $\operatorname{Ord}\left(y_{2}\right)+\operatorname{Ord}\left(y_{3}\right)$. But then $c_{\left(y_{3}\right)_{l}+\left(z_{2}\right)_{l}}$ divides $\operatorname{Ord}\left(x_{1}\right)-\operatorname{Ord}\left(z_{1}\right)$ and $\operatorname{Ord}\left(x_{2}\right)-\operatorname{Ord}\left(z_{1}\right)$, which shows that $\operatorname{Ord}\left(x_{1}\right)>\operatorname{Ord}\left(x_{2}\right)$, contrary to assumption.

Next, suppose $\operatorname{Ord}\left(x_{1}\right)>\operatorname{Ord}\left(x_{2}\right)$. We show $\operatorname{Ord}\left(z_{2}\right)>\operatorname{Ord}\left(y_{3}\right)$. Find $p$ and $q$ such that $\operatorname{Ord}\left(x_{2}\right)=(p+1) \operatorname{Ord}\left(y_{2}\right)+p \operatorname{Ord}\left(y_{3}\right)$ and $\operatorname{Ord}\left(x_{1}\right)=(q+$ 1) $\operatorname{Ord}\left(z_{1}\right)+q \operatorname{Ord}\left(z_{2}\right)$. In this case, $\operatorname{Ord}\left(y_{2}\right)=q \operatorname{Ord}\left(z_{1}\right)+(q-1) \operatorname{Ord}\left(z_{2}\right)$. Hence, $\operatorname{Ord}\left(x_{2}\right)=(p+1) q \operatorname{Ord}\left(z_{1}\right)+(p+1)(q-1) \operatorname{Ord}\left(z_{2}\right)+p \operatorname{Ord}\left(y_{3}\right)$. By assumption, $(1-p q) \operatorname{Ord}\left(z_{1}\right)+(p-p q+1) \operatorname{Ord}\left(z_{2}\right)-p \operatorname{Ord}\left(y_{3}\right)>0$. Since $p q \geq 1$, this in particular implies that $\operatorname{Ord}\left(z_{2}\right)>\operatorname{Ord}\left(y_{3}\right)$, as desired. 
Lemma 2.10. (i) Suppose $\left(x_{1}, x_{2}, x_{3}\right) \in T P C_{3,1}$ with $\left|x_{1}\right|>\left|x_{2}\right|+\left|x_{3}\right|$ and $\operatorname{Ord}\left(x_{2}\right)<\operatorname{Ord}\left(x_{3}\right)$. Let $\psi\left(x_{2}, x_{3}\right)=\left(y_{2}, y_{3}\right)$ and suppose $\operatorname{Ord}\left(y_{2}\right)<\operatorname{Ord}\left(x_{1}\right)$. Let $\psi\left(x_{1}, y_{2}\right)=\left(z_{1}, z_{2}\right)$. Then $\operatorname{Ord}\left(z_{2}\right)<\operatorname{Ord}\left(y_{3}\right)$.

(ii) Similarly, suppose $\left(x_{1}, x_{2}\right) \in T P C_{2,1}$ with $\left|x_{1}\right|>\left|x_{2}\right|$ and $x_{2} \in E P T P^{r}$. Then set $\psi^{r}\left(x_{2}\right)=\left(y_{2}, y_{3}\right)$ and suppose $\operatorname{Ord}\left(y_{2}\right)<\operatorname{Ord}\left(x_{1}\right)$. Setting $\psi\left(x_{1}, y_{2}\right)=$ $\left(z_{1}, z_{2}\right)$, it follows that $\operatorname{Ord}\left(z_{2}\right)<\operatorname{Ord}\left(y_{3}\right)$.

Proof. Again, parts (i) and (ii) have nearly the same proof, which follows. Suppose, on the contrary, that $\operatorname{Ord}\left(z_{2}\right) \geq \operatorname{Ord}\left(y_{3}\right)$. Clearly $\operatorname{Ord}\left(z_{2}\right) \neq \operatorname{Ord}\left(y_{3}\right)$, else $y_{3}$ would have reversed orientation, which is not possible by Lemma 2.4. So $\operatorname{Ord}\left(z_{2}\right)>\operatorname{Ord}\left(y_{3}\right)$. Now, $T^{\operatorname{Ord}\left(x_{1}\right)}$ is defined on $\left(y_{3}\right)_{l}$ since, in case (i), $T^{\operatorname{Ord}\left(x_{1}\right)}$ is defined on $\left(x_{2}\right)_{h}$ and $\left(x_{3}\right)_{h}$, which follows from $\left|x_{1}\right|>\left|x_{2}\right|+\left|x_{3}\right|$, and in case (ii), $T^{\operatorname{Ord}\left(x_{1}\right)}$ is defined on $\left(x_{2}\right)_{h}$. In particular, $T^{\operatorname{Ord}\left(z_{2}\right)}$ is defined on $\left(y_{3}\right)_{l}$. But now, $T^{-\operatorname{Ord}\left(z_{1}\right)}\left(\left(y_{3}\right)_{l}+\left(z_{2}\right)_{l}\right) \overline{<} T^{\operatorname{Ord}\left(y_{3}\right)}\left(\left(y_{3}\right)_{l}+\left(z_{2}\right)_{l}\right)$ but $T^{\operatorname{Ord}\left(z_{2}\right)}\left(\left(y_{3}\right)_{l}+\left(z_{2}\right)_{l}\right) \lessdot T^{\operatorname{Ord}\left(y_{3}\right)}\left(\left(y_{3}\right)_{l}+\left(z_{2}\right)_{l}\right)$, contradicting Lemma 2.6.

Lemma 2.11. Suppose $x=\left(x_{1}, x_{2}, x_{3}, x_{4}\right) \in T P C_{4,2}^{o}$ with $\left|x_{2}\right|<\left|x_{3}\right|+\left|x_{4}\right|$ and $\left|x_{3}\right|<\left|x_{2}\right|+\left|x_{1}\right|$. Let $\psi\left(x_{1}, x_{2}\right)=\left(y_{1}, y_{2}\right)$ and $\psi\left(x_{3}, x_{4}\right)=\left(y_{3}, y_{4}\right)$. Then $\left|x_{2}\right|>\left|x_{3}\right|$ iff $\left(y_{1}, y_{2}, x_{3}, x_{4}\right) \in T P C^{o}$ and $\left|x_{3}\right|>\left|x_{2}\right|$ iff $\left(x_{1}, x_{2}, y_{3}, y_{4}\right) \in T P C^{o}$.

Proof. First we note that, if $\left|x_{2}\right|>\left|x_{3}\right|$, then $\left(y_{1}, y_{2}, x_{3}, x_{4}\right) \in T P C^{o}$, for the following reason. Suppose $\left|x_{2}\right|>\left|x_{3}\right|$ and write $\phi\left(x_{2}, x_{3}\right)=\left(z_{2}, z_{3}\right)$ or $\phi\left(x_{2}, x_{3}\right)=$ $z_{3}$, depending on whether $\left(x_{2}, x_{3}\right)$ is bad or good. Then $\left|z_{2}\right|+\left|z_{3}\right|<\left|x_{4}\right|$, so Lemma 2.10 applies and shows that $\operatorname{Ord}\left(y_{2}\right)<\operatorname{Ord}\left(x_{3}\right)$, as desired. By symmetry, $\left|x_{3}\right|>\left|x_{2}\right|$ implies that $\left(x_{1}, x_{2}, y_{3}, y_{4}\right) \in T P C^{o}$.

So, it suffices to show that $\left|x_{2}\right|>\left|x_{3}\right|$ if $\left(x_{1}, x_{2}, y_{3}, y_{4}\right) \in T P C^{o}$ and $\left|x_{3}\right|>\left|x_{2}\right|$ if $\left(y_{1}, y_{2}, x_{3}, x_{4}\right) \in T P C^{o}$. By the symmetry of the situation, we need only prove the first.

Assume, for sake of contradiction, that $\left|x_{3}\right|>\left|x_{2}\right|$ and $\left(x_{1}, x_{2}, y_{3}, y_{4}\right) \in$ $T P C^{o}$. First suppose that $\left|x_{1}\right|+\left|x_{2}\right|>\left|x_{3}\right|+\left|x_{4}\right|$. Then we note that $T^{-\operatorname{Ord}\left(x_{2}\right)}$ is defined on $\left(y_{4}\right)_{l}$ because it is defined on $\left(x_{3}\right)_{l}$ and $\left(x_{4}\right)_{l}$. Next we note that $T^{-\operatorname{Ord}\left(x_{2}\right)}\left(y_{4}\right)_{l}>T^{-\operatorname{Ord}\left(y_{3}\right)}\left(y_{4}\right)_{l}$ because $\left|y_{4}\right|>\left|y_{3}\right|+\left|x_{2}\right|$. Also, $T^{-\operatorname{Ord}\left(x_{2}\right)}\left(y_{4}\right)_{l} \not \not L$ $\left(y_{4}\right)_{h}$, and $T^{-\operatorname{Ord}\left(y_{3}\right)}\left(y_{4}\right)_{l}<\left(y_{4}\right)_{h}$. This contradicts Lemma 2.6.

On the other hand, it is impossible that $\left|x_{3}\right|+\left|x_{4}\right|>\left|x_{1}\right|+\left|x_{2}\right|$. If this were true, then it would follow that $T^{\operatorname{Ord}\left(x_{2}\right)+\operatorname{Ord}\left(x_{3}\right)}$ shifted $T^{-\operatorname{Ord}\left(x_{2}\right)}\left(\left(x_{1}\right)_{h}+\left(x_{2}\right)_{h}\right)$ to the right by $\left|x_{3}\right|-\left|x_{2}\right|<\left|x_{1}\right|$, while $x_{1} \prec T^{-\operatorname{Ord}\left(x_{2}\right)}\left(\left(x_{1}\right)_{h}\right)$. This would contradict Corollary 2.2.

Lemma 2.12. Suppose $\left(x_{1}, x_{2}\right) \in T P C_{2,1}$ and $\left|x_{1}\right|>\left|x_{2}\right|$. Suppose further that $x_{1} \in P T P^{l}$ and $\psi^{l}\left(x_{1}\right)=\left(y_{1}, y_{2}\right)$. Then $\operatorname{Ord}\left(y_{2}\right)<\operatorname{Ord}\left(x_{2}\right)$.

Proof. Clearly $\operatorname{Ord}\left(y_{2}\right)=\operatorname{Ord}\left(x_{2}\right)$ would imply that $y_{2}, x_{2}$, and $x_{1}$ reverse orientation, which is not possible since $\left|x_{1}\right|>\left|x_{2}\right|$. Suppose instead that $\operatorname{Ord}\left(y_{2}\right)>$ $\operatorname{Ord}\left(x_{2}\right)$. We have that $T^{\operatorname{Ord}\left(x_{1}\right)}$, and hence $T^{\operatorname{Ord}\left(y_{2}\right)}$, is defined on $\left(x_{2}\right)_{h}$, and hence $\left(x_{2}\right)_{l}$. Since $\operatorname{Ord}\left(y_{2}\right)>\operatorname{Ord}\left(x_{2}\right)$, we find that $T^{-\operatorname{Ord}\left(y_{1}\right)}\left[\left(x_{2}\right)_{l}+\right.$ $\left.\left(y_{2}\right)_{l}\right]<T^{\operatorname{Ord}\left(x_{2}\right)}\left[\left(x_{2}\right)_{l}+\left(y_{2}\right)_{l}\right]>T^{\operatorname{Ord}\left(y_{2}\right)}\left[\left(x_{2}\right)_{l}+\left(y_{2}\right)_{l}\right]$, contradicting Lemma 2.6. So $\operatorname{Ord}\left(y_{2}\right)<\operatorname{Ord}\left(x_{2}\right)$. 
Lemma 2.13. Suppose $x=\left(x_{1}, x_{2}, x_{3}\right) \in T P C_{3,2}^{o}$ with $\left|x_{2}\right|<\left|x_{3}\right|<\left|x_{1}\right|+\left|x_{2}\right|$. Then $x_{2} \notin P T P^{l}$ and $x_{3} \notin E P T P^{r}$.

Proof. First, note that $T^{-\operatorname{Ord}\left(x_{2}\right)}$ is defined on $\left(x_{3}\right)_{l}$ since it is defined on $\left(x_{2}\right)_{h}+$ $\left(x_{1}\right)_{h}$, and the former is a subset of the latter on the diagram. If $x_{2} \in E P T P^{l}$, then set $q=P O^{l}\left(x_{2}\right)$. If $x_{3} \in E P T P^{r}$ and $x_{2} \notin E P T P^{l}$, set $q=\operatorname{Ord}\left(x_{2}\right)+$ $\operatorname{Ord}\left(x_{3}\right)-P O^{r}\left(x_{3}\right)$. In either case, $T^{q}$ is defined on $T^{-\operatorname{Ord}\left(x_{2}\right)}\left(\left(x_{3}\right)_{l}\right)$. Indeed, $T^{\operatorname{Ord}\left(x_{2}\right)+\operatorname{Ord}\left(x_{3}\right)}$ is defined on $T^{-\operatorname{Ord}\left(x_{2}\right)}\left(\left(x_{3}\right)_{l}\right)$, and $q<\operatorname{Ord}\left(x_{2}\right)+\operatorname{Ord}\left(x_{3}\right)$. Note that, by Lemma 2.4, $x_{3}$ and $x_{2}$ must preserve orientation. Hence, $T^{q}$ and $T^{\operatorname{Ord}\left(x_{2}\right)+\operatorname{Ord}\left(x_{3}\right)}$ must preserve orientation on $T^{-\operatorname{Ord}\left(x_{2}\right)}\left(\left(x_{3}\right)_{l}\right)$. Set $\omega=$ $T^{-\operatorname{Ord}\left(x_{2}\right)}\left(\left(x_{3}\right)_{l}\right)$. We have $\omega>T^{q} \omega \lessdot T^{\operatorname{Ord}\left(x_{2}\right)+\operatorname{Ord}\left(x_{3}\right)} \omega=\left(x_{3}\right)_{h}$, contradicting Lemma 2.6.

Now, we are ready to define maps $\Phi, \Psi_{ \pm}, \Psi^{r}, \Psi^{l}, \Psi^{\prime}$, and $\Phi^{\prime}$ which parameterize terms which cancel in the expansion of $J^{-1} R_{s t} J^{21}$ (given in Corollary $2.4)$.

Notation 2.2. For any map $f$ taking two arguments, let $f^{i, j}$ be the map $f$ applied to the $i$ and $j$-th components of some larger $k$-tuple. Similarly, for any map $g$ taking only one argument, define $g^{(i)}$ to be the map $g$ applied to the $i$-th component of a larger $k$-tuple.

Definition 2.24. Define $\Phi: T P C_{n, m}^{o} \rightarrow T P C$ for $1 \leq m<n$ as follows. For $x=\left(x_{1}, \ldots, x_{n}\right) \in T P C_{n, m}^{o}$, let $\Phi(x)=\phi^{m, m+1}(x)$.

Definition 2.25. Define $\Psi_{+}: T P C_{n, m}^{o} \rightarrow T P C_{n, m-1}$ for $m \geq 2$ by $\Psi_{+}(x)=$ $\psi^{m-1, m}(x)$. For $m \leq n-2$, define $\Psi_{-}: T P C_{n, m}^{o} \rightarrow T P C_{n, m+1}$ by $\Psi_{-}(x)=$ $\psi^{m+1, m+2}(x)$.

Note 2.8. Note that, unlike in the case of the map $\psi$ (i.e. the case of $2, m$ chains), we can have chains on which both maps $\Psi_{+}$and $\Psi_{-}$are defined. We see, however, that in many cases (namely, the hypotheses of Lemma 2.11), only one map will yield an outer chain.

Definition 2.26. When $x=\left(x_{1}, \ldots, x_{n}\right) \in T P C_{n, m}$ and $x_{m} \in P T P^{l}, x$ is said to be left-passed and we write $x \in L P C$. If $x=\left(x_{1}, \ldots, x_{n}\right) \in T P C_{n, m}$ and $x_{m+1} \in P T P^{r}$ or HPTP $P^{r}$, then $x$ is said to be right-passed or half-passed, and $x \in R P C$ or $H P C$, respectively.

Note 2.9. Note that, given $x=\left(x_{1}, \ldots, x_{n}\right) \in T P C_{n, m}$ with $n \geq m+1 \geq 2$, $x_{m} \in H P T P^{l}$ iff $x_{m+1} \in H P T P^{r}$, so in these cases the half-passed chains not only have a half right-passed pair but also a half left-passed pair.

Definition 2.27. Define $\Psi^{l}: T P C_{n, m}^{o} \cap L P C \rightarrow T P C_{n+1, m}$ by $\Psi^{l}(x)=$ $\left(\psi^{l}\right)^{(m)}(x)$ Similarly, define $\Psi^{r}: T P C_{n, m}^{o} \cap(R P C \cup H P C) \rightarrow T P C_{n+1, m+1}$ by $\Psi^{r}(x)=\left(\psi^{r}\right)^{(m+1)}(x)$.

As in the case of $\phi, \psi, \psi^{*}$, we have the following: 
Lemma 2.14. Let $x, y \in T P C^{o}$. Then $x=\Phi(y)$ iff $y=\Psi_{ \pm}(x)$ for some sign \pm or $y=\Psi^{*}(x)$ for some superscript $* \in\{l, r\}$.

Proof. This follows easily from Proposition 2.1.

Definition 2.28. A chain $x=\left(x_{1}, \ldots, x_{n}\right) \in T P C_{n, m}$ is said to be negatively special if $n \geq m+1 \geq 3$ and $\left|x_{m+1}\right|>\left|x_{m}\right|+\left|x_{m-1}\right|$. The set of such chains is denoted $S P C_{-}$. Similarly, $x=\left(x_{1}, \ldots, x_{n}\right)$ is positively special (or in $S P C_{+}$) if $n \geq m+2 \geq 3$ and $\left|x_{m}\right|>\left|x_{m+1}\right|+\left|x_{m+2}\right|$. Set $S P C=S P C_{+} \cup S P C_{-}$; the general term will be simply special.

Definition 2.29. A chain $x=\left(x_{1}, \ldots, x_{n}\right) \in T P C_{n, m}$ is said to be negatively reversed if $3 \leq m+2 \leq n$ and $x_{m}$ and $x_{m+2}$ both reverse orientation. The set of such chains is $R C_{-}$. A chain $x=\left(x_{1}, \ldots, x_{n}\right) \in T P C_{n, m}$ is positively reversed (or in $R C_{+}$) if $3 \leq m+1 \leq n$ and $x_{m-1}$ and $x_{m+1}$ both reverse orientation. As before, $R C=R C_{+} \cup R C_{-}$is the set of reversed chains.

Lemma 2.15. If $x \in T P C_{n, m}^{o} \cap R C$, then $(n, m)=(3,1)$ or $(3,2)$. If $x \in$ $T P C^{o} \cap R C$, then $\Phi(x), \Psi_{ \pm}(x), \Psi^{*}(x) \notin T P C^{o}$ for any, $\pm *$. In fact, if $x \in$ $T P C^{o} \cap R C_{+}$, then $\Psi_{-}$is not defined on $x$, and $\Psi_{+}(x)=\left(y_{1}, y_{2}, y_{3}\right) \in T P C_{3,1}$ satisfies $\operatorname{Ord}\left(y_{2}\right)=\operatorname{Ord}\left(y_{3}\right)$. Additionally, $\Phi(x)=\left(z_{1}, z_{2}, z_{3}\right) \in T P C_{3,3}$ or $\Phi(x)=\left(z_{1}, z_{2}\right) \in T P C_{2,2}$ where $\operatorname{Ord}\left(z_{1}\right)=\operatorname{Ord}\left(z_{2}\right)$.

Similarly, if $x \in T P C^{o} \cap R C_{-}$, then $\Psi_{+}$is not defined on $x$, and $\Psi_{-}(x)=$ $\left(y_{1}, y_{2}, y_{3}\right) \in T P C_{3,2}$ satisfies $\operatorname{Ord}\left(y_{1}\right)=\operatorname{Ord}\left(y_{2}\right)$. Additionally, $\Phi(x)=$ $\left(z_{1}, z_{2}, z_{3}\right) \in T P C_{3,0}$ or $\Phi(x)=\left(z_{2}, z_{3}\right) \in T P C_{2,0}$ where $\operatorname{Ord}\left(z_{2}\right)=\operatorname{Ord}\left(z_{3}\right)$.

Proof. This easily follows from Lemmas 2.4 and 2.1 .

Corollary 2.3. No outer chain is both reversed and special.

Proof. Clear.

Definition 2.30. We define maps $\Psi^{\prime}, \Phi^{\prime}: T P C^{o} \cap R C \rightarrow T P C^{o}$ as follows. If $x \in R C_{-} \cap T P C^{o}$ where $\Psi_{-}(x)=\left(y_{1}, y_{2}, y_{3}\right)$, set $\Psi^{\prime}(x)=\left(Q\left(y_{1}, y_{2}\right), y_{3}\right) \in$ $T P C_{2,1}$. If $\Phi(x)=\left(z_{1}, z_{2}, z_{3}\right)$, set $\Phi^{\prime}(x)=\left(z_{1}, Q\left(z_{2}, z_{3}\right)\right) \in T P C_{2,0}$, and if $\Phi(x)=\left(z_{2}, z_{3}\right)$, set $\Phi^{\prime}(x)=\left(Q\left(z_{2}, z_{3}\right)\right) \in T P C_{1,0}$. Similarly, if $x \in R C_{+} \cap T P C^{o}$ where $\Psi_{+}(x)=\left(y_{1}, y_{2}, y_{3}\right)$, set $\Psi^{\prime}(x)=\left(y_{1}, Q\left(y_{2}, y_{3}\right)\right) \in T P C_{2,1}$. If $\Phi(x)=$ $\left(z_{1}, z_{2}, z_{3}\right)$, set $\Phi^{\prime}(x)=\left(Q\left(z_{1}, z_{2}\right), z_{3}\right) \in T P C_{2,2}$, and if $\Phi(x)=\left(z_{1}, z_{2}\right)$, then set $\Phi^{\prime}(x)=\left(Q\left(z_{1}, z_{2}\right)\right) \in T P C_{1,1}$.

Lemma 2.16. ( $i)$ Take $x=\left(x_{1}, \ldots, x_{n}\right) \in T P C_{n, m}^{o} \cap S P C_{ \pm}$for some fixed sign \pm . Then $\Psi_{ \pm}(x) \in T P C^{o}$ or $\Phi(x) \in T P C^{o}$, but not both. In the former case, $\Psi_{ \pm}(x) \in S P C_{ \pm}$as well.

(ii) If $x=\left(x_{1}, \ldots, x_{n}\right) \in T P C_{n, m}^{o} \backslash S P C$, for $(n, m) \notin\{(1,0),(1,1),(2,1)\}$, then either $(a) \Psi_{+}(x) \in T P C^{o},(b) \Psi_{-}(x) \in T P C^{o}$, or $(c)$ neither. In case $(c)$, $x \in R C$. In case $(a), \Psi_{+}(x) \in S P C$, and in case $(b), \Psi_{-}(x) \in S P C$.

(iii) If $x \in T P C_{n, m}^{o} \cap[L P C \cup H P C \cup R P C]$, then $x \in S P C$. Additionally, $\Psi^{l}(x) \in T P C^{o} \cap S P C_{+}$if $x \in S P C_{+} \cap L P C$ and $\Psi^{r}(x) \in T P C^{o} \cap S P C_{-}$if $x \in S P C_{-} \cap[R P C \cup H P C]$. 
Proof. (i) This follows immediately from Lemma 2.9 in the cases that $m \geq 2, x \in$ $S P C_{+}$and $m \leq n-2, x \in S P C_{-}$. (Note that it is impossible to have $x \in R C$ by Corollary 2.3.) In the case that $m=1$ and $x \in S P C_{+}$or in the case that $m=n-1$ and $x \in S P C_{-}$, it is clear that $\Phi(x) \in T P C^{o}$.

(ii) It follows that only one of the $\Psi_{+}(x), \Psi_{-}(x)$ can be in $T P C^{o}$ from Lemma 2.11. If $x \in R C \cap T P C^{o}$, then no $\Psi_{ \pm}(x)$ is outer by Lemma 2.15. By Lemma 2.11 , if $n \geq 4$, there is nothing to prove. If $n=3$ and $x \notin R C$, then Lemma 2.10 gives the desired result. For $n=2$ the result is easy.

(iii) The two statements follow from Lemmas 2.13, 2.12, respectively.

Definition 2.31. We define the following subsets of $T P C^{\circ}$ :

(a) $C_{a}=\left\{x \in S P C \cap T P C^{o} \mid \Phi(x) \notin T P C^{o}\right\}$

(a') $C_{b}=\left\{x \in S P C \cup T P C_{2,1} \mid x, \Phi(x) \in T P C^{o}\right\}$

(b) $C_{c}^{ \pm}=\left\{x \in T P C^{o} \backslash[S P C \cup R C] \mid \Psi_{ \pm}(x) \in T P C^{o}\right\}$

Furthermore, let $C_{a}^{ \pm}=C_{a} \cap S P C_{ \pm}, C_{b}^{ \pm}=C_{b} \cap S P C_{ \pm}$, and $C_{c}=C_{c}^{+} \cup C_{c}^{-}$.

Lemma 2.17. The subsets $C_{a}, C_{b}, C_{c}, R C \cap T P C^{o}, T P C_{1,1}$, and $T P C_{1,0}$ are disjoint and their union is all of $T P C^{\circ}$.

Proof. That $C_{a}, C_{b}$, and $C_{c}$ are disjoint from $R C$ follows from Corollary 2.3, Lemma 2.15, and the definition, respectively. The other facts regarding disjointedness are obvious. To check that the union is all of $T P C^{\circ}$, we apply Lemma 2.16.ii, which shows that $C_{c}=T P C^{o} \backslash\left[S P C \cup R C \cup T P C_{1,1} \cup T P C_{1,0} \cup T P C_{2,1}\right]$. It is clear, though, that $T P C_{2,1} \subset C_{b}$. This proves the desired result.

Lemma 2.18. ( $i)$ For each choice of sign, $\Psi_{ \pm}$maps $C_{a}^{ \pm} \cup C_{c}^{ \pm}$injectively to $C_{b}^{ \pm}$.

(ii) $\Psi^{r}$ maps $\left[C_{a}^{-} \cup C_{b}^{-}\right] \cap[R P C \cup H P C]$ injectively to $C_{b}^{-} \backslash[R P C \cup H P C]$ and $\Psi^{l}$ maps $\left[C_{a}^{+} \cup C_{b}^{+}\right] \cap L P C$ injectively to $C_{b}^{+} \backslash L P C$.

(iii) $C_{c} \cap[L P C \cup R P C \cup H P C]=\emptyset$.

(iv) $\Psi^{r}\left[\left(C_{a}^{+} \cup C_{b}^{+}\right) \cap(R P C \cup H P C)\right]$ and $\Psi^{l}\left[\left(C_{a}^{-} \cup C_{b}^{-}\right) \cap L P C\right]$ are both disjoint from $C_{b}$.

(v) $\Psi_{ \pm}\left(C_{b}\right)$ is disjoint from $T P C^{o}$ for each choice of sign.

Proof. (i) This follows immediately from Lemmas 2.16.i and 2.14.

(ii) This follows immediately from Lemmas 2.16.ii, 2.14, and 2.8.

(iii) This follows immediately from Lemma 2.13.

(iv) This follows immediately from the definition of $S P C$.

(v) This follows from Lemma 2.16.i, since we know $C_{b}$ is disjoint from $R C$.

Corollary 2.4. $T P C^{o}$ is partitioned by all sets of the form:

(a) For $x \in C_{a}^{+} \cup C_{c}^{+}$,

(2.12) $\quad A_{x}=$

$\left\{x, \Psi_{+}(x)\right\} \cup\left\{y \mid y=\Psi^{l}(x)\right.$ or $\left.y=\Psi^{l} \circ \Psi_{+}(x)\right\} \cup\left\{x_{p} \in R C_{+} \mid \Phi^{\prime}\left(x_{p}\right)=x\right\}$, 
$\left(a^{\prime}\right)$ Those of the form (2.12), replacing + with - and $l$ with $r$. (Call these also $A_{x}$, distinguished from the above by the "sign" of $x$.)

(b) For a fixed $x \in T P C_{1,1}$,

$$
\begin{aligned}
& A_{x}= \\
& \quad\{x\} \cup\left\{y \mid y=\Psi^{l}(x) \text { or } y=\Psi^{l} \circ \Psi^{l}(x)\right\} \cup\left\{x_{p} \in R C_{+} \mid \Phi^{\prime}\left(x_{p}\right)=x\right\}
\end{aligned}
$$

(b') Those of form (2.13), replacing + with,$- l$ with $r$, and $T P C_{1,1}$ with $T P C_{1,0}$. Call these $A_{x}$ as well.

Here it is meant that, if $\Psi^{*}$ is not defined on $x$, then $y \neq \Psi^{*}(x)$ for any $y$.

Proof. For every $x \in C_{a}^{+} \cup C_{c}^{+}$, we see from the Lemma, part (i), that $\Psi_{+}(x) \in$ $C_{b} \subset T P C^{o}$. Furthermore, $\Psi^{l}(x) \in C_{b}$ and $\Psi^{l} \circ \Psi_{+}(x) \in C_{b}$ where these are defined (Lemma, part ii). Now take any $y \in C_{b}^{+}$. First note that either $\Psi_{+}(\Phi(y))=y$ or $\Psi^{l}(\Phi(y))=y$ because $y \in S P C_{+}$. Note that $\Phi(y) \notin R C$ because $\Psi_{+}\left(R C_{+}\right)$and $\Psi^{l}\left(R C_{+} \cap L P C\right)$ are disjoint from $T P C^{o}$ (Lemma 2.15). Now, if $\Phi(y) \notin C_{b}$, then using Lemma 2.17, $y \in A_{\Phi(y)}$ (of type a or b, depending on whether $\Psi_{+}(\Phi(y))=y$ or $\Psi^{l}(\Phi(y))=y$, respectively). If $\Phi(y) \in C_{b}$, then clearly $y \in C_{b}^{+}$. In this case, $\Phi^{2}(y) \notin C_{b}$ by the Lemma, parts (ii), (iv), and (v), using Lemma 2.14. Furthermore, in this case $\Psi^{l}\left(\Phi^{2}(y)\right)=\Phi(y)$, again from the Lemma, parts (iv) and (v). Then, $y \in A_{\Phi^{2}(y)}$. Thus, we see that all elements $y \in C_{b}^{+}$fit into at least one of the sets (a) or (b). To see that all elements in $C_{b}^{+}$fit into at most one, note that the way in which $y$ appears is uniquely determined by which of the following holds: (A) $\Phi(y) \notin C_{b}, y=\Psi_{+} \circ \Phi(y)$; (B) $\Phi(y) \notin C_{b}, y=\Psi^{l} \circ \Phi(y)$; (C) $\Phi(y) \in C_{b}, \Phi(y)=\Psi^{l} \circ \Phi^{2}(y)$. Now, it remains to consider those elements of $R C$. For any $z \in R C$, we know that $\Phi^{\prime}(z) \in T P C_{2,2}^{o} \cup T P C_{2,0}^{o} \cup T P C_{1,1}^{o} \cup T P C_{1,0}^{o}$ by Lemma 2.15. The former two are subsets of $C_{c}$, so we know that $z \in A_{\Phi^{\prime}(z)}$ for any $z \in R C$, and clearly $\Phi^{\prime}(z)$ is unique. The negative case is almost identical to this one.

Definition 2.32. For $x \in T P C_{n, m}^{o}$, set $E(x)=1$ if $x_{m} \in H P T P_{l}$ or $x_{m+1} \in$ $H P T P_{r}$ and 0 otherwise. Now, given $x=\left(x_{1}, \ldots, x_{n}\right) \in T P C_{n, m}^{o}$, set $P(x)=$ $(-1)^{m}\left(q-q^{-1}\right)^{n} q^{E(x)} \prod_{i=1}^{n}(-q)^{S_{x_{i}} C_{x_{i}}\left(\left|x_{i}\right|-1\right)} q^{P^{a}\left(x_{i}\right)}$.

Lemma 2.19. $J^{-1} R_{s t} J^{21}=\sum_{x \in T P C^{o}} P(x) E_{Q(x)}+\sum_{i \neq j} e_{i i} \otimes e_{j j}+\sum_{i} q e_{i i} \otimes e_{i i}$.

Proof. The terms in the expansion of $J^{-1} R_{s t} J^{21}$ are

$$
q^{E(x)} \prod_{i=1}^{n} E_{x_{i}} S_{x_{i}}\left(q-q^{-1}\right)(-q)^{-S_{x_{i}} C_{x_{i}}\left(\left|x_{i}\right|-1\right)} q^{P^{a}\left(x_{i}\right)},
$$

for each $x=\left(x_{1}, \ldots, x_{n}\right) \in T P C^{o}$, as well as the terms $e_{i i} \otimes e_{j j}$ for $i \neq j$ and $q e_{i i} \otimes e_{i i}$ for each $i$.

Lemma 2.20. ( $i)$ If $x \in C_{a} \cup C_{c}$, then $\sum_{y \in A_{x}} P(y) E_{Q(y)}=0$. (ii) If $x=\left(x_{1}\right)$ $\in T P C_{1,1} \cup T P C_{1,0}$, then $\sum_{y \in A_{x}} P(y) E_{Q(y)}=-S_{x_{1}} q^{S_{x_{1}}\left[P^{s}\left(x_{1}\right)+C_{x_{1}}\left(\left|x_{1}\right|-1\right)\right.} E_{x_{1}}$. 
Proof. (i) Let $x \in C_{a}^{+} \cup C_{c}^{+}$. The negative case is similar (see comments at the end of the proof of this part). Let $x=\left(x_{1}, \ldots, x_{n}\right) \in T P C_{n, m}$ and let

$$
(-1)^{C_{x_{m-1}}\left(\left|x_{m-1}\right|-1\right)} \prod_{i=1}^{m-2}-(-q)^{C_{x_{i}}\left(\left|x_{i}\right|-1\right)} q^{P^{a}\left(x_{i}\right)} \prod_{j=m+1}^{n}(-q)^{C_{x_{j}}\left(1-\left|x_{j}\right|\right)} q^{P^{a}\left(x_{j}\right)}
$$

be the part of the formula for $P(x)$ which will not change upon applying $\Psi_{+}$, $\Psi^{l}$, and $\Psi^{\prime}$ (where applicable).

Let $B=P\left(\Psi^{l}(x)\right)$ if $x \in L P C$ and $B=0$ otherwise. Set $\left(y_{m-1}, y_{m}\right)=$ $\psi\left(x_{m-1}, x_{m}\right)$. First we show that

$$
P(x)+B=q^{C_{x_{m-1}}\left(\left|x_{m-1}\right|-1\right)+P^{r}\left(x_{m-1}\right)-P^{l}\left(y_{m}\right)} F .
$$

First, by Lemma 2.7,

$$
P^{l}\left(x_{m-1}\right)=P^{l}\left(x_{m}\right)+P^{r}\left(x_{m}\right)+P^{l}\left(y_{m}\right) .
$$

Hence,

$$
P^{a}\left(x_{m-1}\right)+P^{a}\left(x_{m}\right)=P^{r}\left(x_{m-1}\right)-P^{l}\left(y_{m}\right)-2 P^{l}\left(x_{m}\right) .
$$

If $x \notin L P C$, then $B=0$ and $P^{l}\left(x_{m}\right) \in\left\{0, \frac{1}{2}\right\}$. In this case, (2.16) follows from the definition of $E(t)$. If $x \in L P C$, then $P^{l}\left(x_{m}\right)=1$, and (2.17) implies $P^{r}\left(x_{m}\right)=0$. Then Lemma 2.8 shows that $B=\left(q^{2}-1\right) P(x)$ so that the lefthand side of $(2.16)$ is $q^{2+P^{a}\left(x_{m-1}\right)+P^{a}\left(x_{m}\right)+C_{x_{m-1}}\left(\left|x_{m-1}\right|-1\right)} F$. By (2.18), this is the same as the right-hand side.

Next, we show that

$$
\sum_{\Phi^{\prime}(y)=x} P(y)=q^{P^{r}\left(x_{m-1}\right)-P^{l}\left(y_{m}\right)}\left[q^{C_{x_{m-1}}\left(1-\left|x_{m-1}\right|\right)}-q^{C_{x_{m-1}}\left(\left|x_{m-1}\right|-1\right)}\right] F .
$$

Naturally, we may assume that $x_{m-1}$ reverses orientation. Since, in this case, $y_{m}$ also reverses orientation, it must be that $m=n=2$. Suppose now that $x_{1}=\left(e_{i}-e_{j}, e_{l}-e_{i+l-j}\right)$ and $x_{2}=\left(e_{j}-e_{k}, e_{i+l-j}-e_{i+l-k}\right)$. Now, for every $p$ such that $1 \leq p \leq j-i-1$, set $u_{p}=\left(e_{i}-e_{i+p}, e_{l}-e_{l-p}\right), v_{p}=\left(e_{i+p}-\right.$ $\left.e_{k+j-i-p}, e_{l-p}-e_{2 i+p+l-j-k}\right)$, and $w_{p}=\left(e_{k+j-i-p}-e_{k}, e_{2 i+p+l-j-k}-e_{i+l-k}\right)$. Then $t_{p}=\left(u_{p}, v_{p}, w_{p}\right)$ are exactly those reversed chains such that $\Phi^{\prime}\left(t_{p}\right)=x$. Now, we consider the possible passing properties of the $T$-pairs involved. Note first that, since $\operatorname{Ord}\left(u_{p}\right)=\operatorname{Ord}\left(x_{1}\right), P^{r}\left(x_{1}\right)=P^{r}\left(u_{p}\right)$ for all $p$. For the same reason, $P^{l}\left(w_{p}\right)=P^{l}\left(y_{2}\right)$ for all $p$. Next, note that $P^{l}\left(u_{p}\right)=0$ for all $p-$ otherwise, applying $T^{P O^{l}\left(u_{p}\right)}$ to $x_{1}$ would contradict nilpotency. For the same reason, $P^{r}\left(w_{p}\right)=0$ for all $p$. Next, note that $P^{l}\left(v_{p}\right)=P^{r}\left(v_{p}\right)=0$ for all $p$. This follows from the fact that $P^{l}\left(u_{p}\right)=0$ for all $p$, making use of Lemma 2.7. Now, (2.19) follows readily.

Let $D=P\left(\Psi^{l} \circ \Psi_{+}(x)\right)$ when $\Psi_{+}(x) \in L P C$ and $D=0$ otherwise. Finally, we show that

$$
P\left(\Psi_{+}(x)\right)+D=-q^{C_{x_{m-1}}\left(1-\left|x_{m-1}\right|\right)+P^{r}\left(x_{m-1}\right)-P^{l}\left(y_{m}\right)} F .
$$


The proof is similar to the proof two paragraphs back. If $\Psi_{+}(x) \notin L P C$, then $D=0$. By Lemma 2.7, $P^{r}\left(x_{m-1}\right)=P^{r}\left(y_{m}\right)+P^{r}\left(y_{m-1}\right)+P^{l}\left(y_{m-1}\right)$. Thus $P^{a}\left(y_{m-1}\right)+P^{a}\left(y_{m}\right)+2 P^{l}\left(y_{m-1}\right)=P^{r}\left(x_{m-1}\right)-P^{l}\left(y_{m}\right)$, proving $(2.20)$ in this case (note that $P^{l}\left(y_{m-1}\right) \in\left\{0, \frac{1}{2}\right\}$ and recall the definition of $E(t)$.) If $\Psi_{+}(x) \in L P C$, then $P^{l}\left(y_{m-1}\right)=1$ and by Lemma 2.7 (or the equation above), $P^{r}\left(y_{m-1}\right)=0$ and $P^{a}\left(y_{m-1}\right)+P^{a}\left(y_{m}\right)+2=P^{r}\left(x_{m-1}\right)-P^{l}\left(y_{m}\right)$. If we set $\psi^{l}\left(y_{m-1}\right)=\left(z_{m-1}, z_{m}\right)$, then it follows from Lemma 2.8 that neither $z_{m-1}$ nor $z_{m}$ is passed, so $P^{a}\left(z_{m-1}\right)=P^{a}\left(z_{m}\right)=0$. Hence, $D=\left(q^{2}-1\right) P\left(\Psi_{+}(x)\right)$, so the

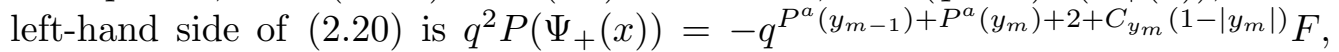
which is equal to the right-hand side by Corollary 2.1 and the above analysis.

Now, putting (2.16), (2.19), and (2.20) together, we get

$$
P(x)+P\left(\Psi_{+}(x)\right)+B+D+\sum_{\Phi^{\prime}(y)=x} P(y)=0,
$$

as desired.

The negative case is almost the same as the above, except that the "error term" $E(t)$ over-corrects, but this is counteracted by the fact that now $\Psi^{r}$ is defined on $H P C$ as well as $R P C$. (The details are omitted.)

(ii) This is almost the same as the proof above. First we note that the result is clear when $x \notin L P C \cup R P C \cup H P C$, as $A_{x}=\{x\}$ and $P(x)$ gives the desired formula (bearing in mind the definition of $E$ ). Suppose $x=\left(x_{1}\right) \in$ $T P C_{1,1} \cap L P C$. Let $B=P\left(\Psi^{l}(x)\right)$ and let $D=P\left(\Psi^{l} \circ \Psi^{l}(x)\right)$ if $x \in L P C$ and $\Psi^{l} \in L P C$, and $D=0$ otherwise. Let $F=-(-1)^{C_{x_{1}}\left(1-\left|x_{1}\right|\right)}\left(q-q^{-1}\right)$. Set $\left(y_{1}, y_{2}\right)=\psi^{l}\left(x_{1}\right)$. First we show that

$$
B+D=\left[q^{C_{x_{1}}\left(1-\left|x_{1}\right|\right)+P^{r}\left(x_{1}\right)+1}-q^{C_{x_{1}}\left(1-\left|x_{1}\right|\right)+P^{r}\left(x_{1}\right)-1}\right] F .
$$

Lemma 2.8 shows that $P^{l}\left(y_{2}\right)=0$ and

$$
P^{r}\left(x_{1}\right)=P^{r}\left(y_{2}\right)+P^{a}\left(y_{1}\right)+2 P^{l}\left(y_{1}\right) .
$$

First suppose $D=0$. In this case, (2.23) implies (2.22) easily. If $D \neq 0$, then $P^{r}\left(x_{1}\right)=P^{l}\left(x_{1}\right)=P^{l}\left(y_{1}\right)=1$, and $P^{r}\left(y_{1}\right)=P^{r}\left(y_{2}\right)=0$. Then, $D=$ $\left(q-q^{-1}\right)^{2} q^{C_{x_{1}}\left(1-\left|x_{1}\right|\right)} F$. Furthermore, $B=\left(1-q^{-2}\right) q^{C_{x_{1}}\left(1-\left|x_{1}\right|\right)} F$. From this (2.22) follows.

Next, we claim that

$$
\sum_{\Phi^{\prime}(y)=x} P(y)=\left(q-q^{-1}\right) q^{P^{r}\left(x_{1}\right)-P^{l}\left(y_{2}\right)}\left[q^{C_{x_{1}}\left(\left|x_{1}\right|-1\right)}-q^{C_{x_{1}}\left(1-\left|x_{1}\right|\right)}\right] F .
$$

This follows by exactly the same arguments as used in the second paragraph of the proof of part (i). But, $P^{l}\left(y_{2}\right)=0$, so, putting (2.22) and (2.24) together, the result easily follows. The negative $/ T P C_{1,0}$ case is almost the same as this, bearing in mind the final comments in the proof of the previous part.

Proof of the main theorem, 1.2. This follows immediately from Lemmas 2.19 and 2.20 and Corollary 2.4, using (1.9) for $R_{\mathrm{GGS}}$. 


\section{Acknowledgements}

I would like to thank Pavel Etingof for introducing me to this problem and advising me. I would also like to thank the Harvard College Research Program for their support. Finally, I am indebted to Gerstenhaber, Giaquinto, and Hodges for valuable discussions and for sharing some unpublished results.

\section{Appendix A. Proof of Proposition 1.4}

In this section we explicitly compute $R_{\mathrm{GGS}}$ for generalized Cremmer-Gervais triples, the only triples satisfying $\left|\Gamma_{1}\right|+1=|\Gamma|$ (omitting only one root), thereby proving Giaquinto's formula, (1.10), and thus Proposition 1.4. Recall from Example 1.1 the results given in [GG]: these triples are indexed by $(n, m)$ where $n=|\Gamma|+1$ is the dimension, $m$ is relatively prime to $n, \Gamma_{1}=\Gamma \backslash\left\{\alpha_{n-m}\right\}$, $\Gamma_{2}=\Gamma \backslash\left\{\alpha_{m}\right\}$, and $T \alpha_{i}=\alpha_{\operatorname{Res}(i+m)}$, where Res give the residue $\bmod n$ in $\{1, \ldots, n\}$. Moreover when $s$ is taken to have trace zero in the first component, it is uniquely given by $s_{i i}^{i i}=0$ and $s_{i j}^{i j}=\frac{1}{2}-\frac{1}{n} \operatorname{Res}\left(\frac{j-i}{m}\right)$ where $i \neq j$. Then, the only difficulty is in computing $q^{s} \tilde{a} q^{s}$, so here we use (1.9) to prove (1.10).

Clearly we have

$$
q^{s} \tilde{a} q^{s}=\sum_{x \in T P_{+}} q^{-P^{s}(x)+r(x)} E_{x}^{21}-q^{P^{s}(x)-r(x)} E_{x},
$$

where $r\left(e_{k}-e_{i+k-j}, e_{i}-e_{j}\right)=s_{j, i+k-j}^{j, i+k-j}+s_{i, k}^{i, k}$, for $j<i$, since $C_{x}=0$ for all $x$. It suffices, then, to show $P^{s}(x)-r(x)=\frac{2 \operatorname{Ord}(x)}{n}$ for all $x$. Take $x=$ $\left(e_{k}-e_{i+k-j}, e_{i}-e_{j}\right)$. Below we use the notation [statement] $=1$ if "statement" is true and 0 if it is false.

One sees that

$$
\begin{aligned}
s_{j, i+k-j}^{j, i+k-j}+s_{i, k}^{i, k}= & 1-\frac{1}{2}[2 j=i+k]-\frac{1}{2}[i=k]- \\
& \frac{1}{n} \operatorname{Res}\left(\frac{i+k-2 j}{m}\right)-\frac{1}{n} \operatorname{Res}\left(\frac{k-i}{m}\right) \\
= & 1-\frac{1}{2}[2 j=i+k]-\frac{1}{2}[i=k]-\frac{2}{n} \operatorname{Res}\left(\frac{k-j}{m}\right)+M_{i, j, k},
\end{aligned}
$$

where

$$
\begin{aligned}
M_{i, j, k}=[2 j \neq i+k]\left[\operatorname{Res}\left(\frac{k-j}{m}\right)\right. & \left.>\operatorname{Res}\left(\frac{i+k-2 j}{m}\right)\right] \\
& -[i \neq k]\left[\operatorname{Res}\left(\frac{k-j}{m}\right)<\operatorname{Res}\left(\frac{k-i}{m}\right)\right] .
\end{aligned}
$$

Thus, since $\operatorname{Res}\left(\frac{k-j}{m}\right)=\operatorname{Ord}(x)$, it suffices to show $\frac{1}{2}[2 j=i+k]+\frac{1}{2}[i=$ $k]+P^{r}(x)+P^{l}(x)=1+M_{i, j, k}$. Note that $i=k$ iff $x \in H P T P^{r}$ and $2 j=i+k$ iff $x \in H P T P^{l}$, and in these cases $M_{i, j, k}=0$, so it suffices to consider $x \notin H P T P$. 
In this case we need to show $1+M_{i, j, k}=P^{r}(x)+P^{l}(x)$. Now,

(A.4) $1+M_{i, j, k}=$

$$
\left[\operatorname{Res}\left(\frac{k-j}{m}\right)>\operatorname{Res}\left(\frac{i+k-2 j}{m}\right)\right]+\left[\operatorname{Res}\left(\frac{k-j}{m}\right)>\operatorname{Res}\left(\frac{k-i}{m}\right)\right],
$$

and it is not difficult to see that $\left[\operatorname{Res}\left(\frac{k-j}{m}\right)>\operatorname{Res}\left(\frac{i+k-2 j}{m}\right)\right]=P^{l}(x)$ while $\left[\operatorname{Res}\left(\frac{k-j}{m}\right)>\operatorname{Res}\left(\frac{k-i}{m}\right)\right]=P^{r}(x)$. This finishes the proof.

\section{References}

[BD] A. A. Belavin and V. G. Drinfeld, Triangle equations and simple Lie algebras, Soviet Sci. Rev. Sect. C: Math. Phys. Rev. 4 (1984), 93-165.

[ESS] P. Etingof, T. Schedler, and O. Schiffmann, Quantization of dynamical r-matrices, to appear.

[GG] M. Gerstenhaber and A. Giaquinto, Boundary solutions of the classical Yang-Baxter equation, Lett. Math. Phys. 40 (1997), 337-353.

[GGS] M. Gerstenhaber and A. Giaquinto, and S. D. Schack, Construction of quantum groups from Belavin-Drinfeld infinitesimals, Israel Math. Conf. Proc. 7 (1993), 45-64.

[GH] A. Giaquinto and T. J. Hodges, Nonstandard solutions of the Yang-Baxter equation, Lett. Math. Phys. 44 (1998), 67-75.

[H] T. J. Hodges, Nonstandard quantum groups associated to certain Belavin-Drinfeld triples, Contemp. Math. 214 (1998), 63-70.

[H2] , The Cremmer-Gervais solutions of the Yang-Baxter equation, Proc. Amer. Math. Soc. 127 (1999), 1819-1826, q-alg/9712036.

[S] T. Schedler, Verification of the GGS conjecture for $\mathfrak{s l}(n), n \leq 12$, math.QA/9901079.

[S2] , On the GGS conjecture, math.QA/003079.

059 Pforzheimer House Mail Center, Cambridge, MA 02138.

E-mail address: schedler@fas.harvard.edu 